\title{
Demand-side solutions to climate change mitigation consistent with high levels of well-being
}

\author{
Felix Creutzig $\mathbb{1}^{1,2,26 \bowtie}$, Leila Niamir ${ }^{1,26}$, Xuemei Bai ${ }^{(13}{ }^{3}$, Max Callaghan ${ }^{1}{ }^{1,4}$, Jonathan Cullen ${ }^{5}$, \\ Julio Díaz-José ${ }^{6}{ }^{6}$, Maria Figueroa7, Arnulf Grubler ${ }^{8}$, William F. Lamb ${ }^{1,4}$, Adrian Leip ${ }^{9}$, \\ Eric Masanet ${ }^{10}$, Érika Mata11, Linus Mattauch ${ }^{2,12,13}$, Jan C. Minx ${ }^{1,4}$, Sebastian Mirasgedis ${ }^{14}$, \\ Yacob Mulugetta ${ }^{15}$, Sudarmanto Budi Nugroho ${ }^{16}{ }^{16}$, Minal Pathak ${ }^{17}$, Patricia Perkins ${ }^{18}$, \\ Joyashree Roy ${ }^{19,20}$, Stephane de la Rue du Can (1021, Yamina Saheb 22,23, Shreya Some (17,20, \\ Linda Steg ${ }^{24}$, Julia Steinberger ${ }^{23}$ and Diana Ürge-Vorsatz ${ }^{25}$
}

\begin{abstract}
Mitigation solutions are often evaluated in terms of costs and greenhouse gas reduction potentials, missing out on the consideration of direct effects on human well-being. Here, we systematically assess the mitigation potential of demand-side options categorized into avoid, shift and improve, and their human well-being links. We show that these options, bridging socio-behavioural, infrastructural and technological domains, can reduce counterfactual sectoral emissions by $40-80 \%$ in end-use sectors. Based on expert judgement and an extensive literature database, we evaluate 306 combinations of well-being outcomes and demand-side options, finding largely beneficial effects in improvement in well-being (79\% positive, $18 \%$ neutral and 3\% negative), even though we find low confidence on the social dimensions of well-being. Implementing such nuanced solutions is based axiomatically on an understanding of malleable rather than fixed preferences, and procedurally on changing infrastructures and choice architectures. Results demonstrate the high mitigation potential of demand-side mitigation options that are synergistic with well-being.
\end{abstract}

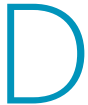
emand-side mitigation options are increasingly discussed in the literature-for example, refs. ${ }^{1-3}$. However, a consistent evaluation in terms of both their overall potential and societal implications is lacking. Even for an ambitious $1.5^{\circ} \mathrm{C}$ target, several mitigation strategies are plausible, ranging from high dependence on new energy infrastructures to low-demand pathways ${ }^{4}$. Evaluation of these options, mostly from a macroeconomic cost-benefit perspective, is relevant but it fails to reflect the wider impacts through benefits and costs of mitigation strategies from the human well-being perspective ${ }^{5}$.

There are three closely related shortcomings. First, mitigation options on the demand side-such as choices toward transport mode for mobility patterns and building design, size and useinteract with the well-being of end users and citizens. Evaluation of the marginal monetary costs of these measures, if they can be monetized at all, hardly reflects their full impacts. Second, a focus on costs leads to a tendency to preferably evaluate those solutions that have precise direct market cost values attached, neglecting more systemic or uncertain solutions where market prices are difficult to evaluate or not relevant ${ }^{6}$. Third, income and expenditures reflect only a part of well-being, and monetary cost evaluations, even if starting from a broader framework, often ignore encompassing views on multiple dimensions of well-being. This critique is not new and, on the aggregate scale, there is agreement among economists and philosophers, and in other disciplines, that metrics such as gross domestic product (GDP) insufficiently reflect well-being, and that these must be complemented by more encompassing metrics?

These considerations motivate two related questions: first, what is the climate change mitigation potential of demand-side mitigation options? Second, what are the implications for well-being of these demand-side mitigation options? In particular, answering the second question is a considerable challenge because there is no single straightforward and agreed on metric of well-being. Well-being can be considered at both the macro level-for example, in ten country-level well-being domains by the Organisation for Economic Co-operation and Development ${ }^{8}$-and at the micro level,

\footnotetext{
${ }^{1}$ Mercator Research Institute on Global Commons and Climate Change, Berlin, Germany. ${ }^{2}$ Technische Universität Berlin, Berlin, Germany. ${ }^{3}$ Australian National University, Canberra, Australian Capital Territory, Australia. ${ }^{4}$ Priestley International Centre for Climate, School of Earth and Environment, University of Leeds, Leeds, UK. ${ }^{5}$ University of Cambridge, Cambridge, UK. ${ }^{6}$ Universidad Veracruzana, Veracruz, Mexico. ${ }^{7}$ Copenhagen Business School, Copenhagen, Denmark. ${ }^{8}$ International Institute for Applied Systems Analysis, Laxenburg, Austria. ${ }^{9}$ European Commission, Joint Research Centre, Ispra, Italy. ${ }^{10}$ University of California, Santa Barbara, CA, USA. ${ }^{11} \mathrm{IVL}$ Swedish Environmental Research Institute, Gothenburg, Sweden. ${ }^{12}$ Potsdam Institute for Climate Impact Research, Potsdam, Germany. ${ }^{13}$ University of Oxford, Oxford, UK. ${ }^{14}$ National Observatory of Athens, Athens, Greece. ${ }^{15}$ University College London, London, UK. ${ }^{16}$ Institute for Global Environmental Strategies, Kanagawa, Japan. ${ }^{17}$ Ahmedabad University, Ahmedabad, India. ${ }^{18}$ York University, Toronto, Ontario, Canada. ${ }^{19}$ Asian Institute of Technology, Bangkok, Thailand. ${ }^{20}$ Jadavpur University, Kolkata, India. ${ }^{21}$ Lawrence Berkeley National Laboratory, Berkeley, CA, USA. ${ }^{22}$ OpenExp, Paris, France. ${ }^{23}$ Faculty of Geosciences and Environment, University of Lausanne, Lausanne, Switzerland. ${ }^{24}$ University of Groningen, Groningen, the Netherlands. ${ }^{25}$ Central European University, Budapest, Hungary. ${ }^{26}$ These authors contributed equally: Felix Creutzig, Leila Niamir凶e-mail: creutzig@mcc-berlin.net
} 
reflected, for example, by individual constituents of well-being ${ }^{9-11}$. Approaches can also be separated into subjective understandings of well-being (given preferences, happiness) and objective ones (life expectancy, eudaimonic metrics) with diverging implications for climate change mitigation ${ }^{12,13}$. According to some leading eudaimonic approaches, well-being has several constituents and all of these must be met independently to enable a good life $\mathrm{e}^{14,15}$.

Here, we follow an eudaimonic understanding and examine the individual metrics and constituents of well-being. We first identify demand-side mitigation options and group these into avoid, shift and improve (ASI) categories for all end-use sectors ${ }^{3}$. We estimate their respective potentials across sectors, informed by a comprehensive literature review (Methods). Then we ask how these improve or harm individual constituents of well-being (Extended Data Fig. 1), systematically coding their impact on constituents of well-being based on a literature review (Methods).

\section{Demand-side options to reduce emissions across sectors}

Following an established definition ${ }^{3}$, demand-side solutions for mitigation of climate change modify demand for goods and services by targeting choices/adoption of technology, consumption, behaviour, lifestyles, coupled production-consumption infrastructures and systems, service provision and associated socio-technical transitions, as exemplified by options to improve accessibility and living conditions and increase nutritional quality while decreasing energy input and emissions of greenhouse gases (GHG). These are distinct from supply-side options that involve changes in energy supply, production technologies and deployment of carbon dioxide-removal technologies that keep demand by end users invariant.

Demand-side options can be grouped into ASI categories, constituting a simple analytical framework pertinent for decision makers ${ }^{3}$. Originally applied to the transport sector ${ }^{16,17}$, these categories can also be transferred to other sectors ${ }^{3,18,19}$. However, a comprehensive bottom-up assessment of ASI options is missing. Here, we generalize 'avoid' to denote all mitigation options that reduce unnecessary consumption (that is, energy or food consumption in developed countries that is not needed for maintenance or improvement of the levels of services provided) by redesigning service-provisioning systems; 'shift' to describe the switch to already existing competitive low-carbon technologies and service-provisioning systems; and 'improve' to mean improvements in efficiency in existing technologies where adoption by end users plays an important role.

A high potential for mitigation is offerred by ASI options (Fig. 1 and Table 1). In all sectors, end-use strategies can help reduce the majority of emissions, ranging from $41 \%$ (6.5 gigatonnes of $\mathrm{CO}_{2}$ equivalent $\left(\mathrm{GtCO}_{2} \mathrm{e}\right)$ ) emission reductions in the industry sector to $41 \%\left(7.3 \mathrm{GtCO}_{2} \mathrm{e}\right)$ in the food sector, to $62 \%\left(5.8 \mathrm{GtCO}_{2} \mathrm{e}\right)$ emission reductions in the land transport sector and $78 \%\left(6.8 \mathrm{GtCO}_{2} \mathrm{e}\right)$ in the buildings sector. These numbers are median estimates and represent benchmark accounting. Estimates are approximations, as they are simple products of individual assessments for each of the three ASI options. When interactions are taken into account, the full mitigation potentials may be higher or lower, independent of relevant barriers to realization of the median potential estimates. Demand-side mitigation potentials here are based on opportunities for action available to end users, while not considering supply-side options such as decarbonization of the electricity sector. However, mitigation potentials include technology adoption that reduces carbon intensity-for example, embedded renewable energy in housing and electric vehicles for transport.

We find that improve options contribute most in the buildings, transport and industry sectors. Examples include efficient building envelopes, household appliances, electric cars and more efficient material and energy use in industrial production. Shift measures are most relevant for transport-in particular a modal shift to walking, cycling and shared pooled mobility; and for food-in particular a shift to flexitarian, vegetarian or vegan diets. These are options that require physical and choice infrastructures that support low-carbon choices, such as safe and convenient transit corridors and desirable and affordable meat-free menu options. They also require end users to adopt these choices, individually and socially. Avoid options are relevant in all sectors. Cities play an additional role, as more compact designs and higher accessibility reduce demand for distanced travel and car mobility and also translate into lower average floor size and corresponding heating, cooling and lighting demand. The lifetime extension of products and buildings and more efficient product design also add to avoidance of energy use and related emissions. Teleworking is related to high uncertainty with relatively low mitigation potential in consequential assessments, but with possibly higher GHG emission reduction potential if COVID-19 experiences induce a structural shift in working environments from both employees and employers.

Opportunities for avoiding excess consumption exist for all end-use sectors. Reducing food waste is a prime no-regret option, accounting for $4.4 \mathrm{GtCO}_{2} \mathrm{e}$ emissions, or $8 \%$ of total annual GHG emissions if deforestation effects associated with wasted food provision are included ${ }^{20}$. In developed countries, consumers are the largest source of food waste and habitual adjustments, such as meal planning, reuse of leftovers and avoidance of overpreparation reduce associated GHG emissions ${ }^{21,22}$. Reregulating expiration labels is an option for policy makers to disincentivize unnecessary disposal of unexpired items ${ }^{23}$. The annual mitigation potential of food waste reductions globally by 2050 has been estimated at $0.8-6.0 \mathrm{GtCO}_{2} \mathrm{e}^{24,25}$.

Diet shifts, as another demand-side strategy, are even more impactful in the food sector. Estimated annual GHG emissions reductions by 2050 associated with dietary shifts to low-meat, vegetarian or vegan diets range from $0.7-7.3,4.3-6.4$ and $7.8-8 \mathrm{GtCO}_{2} \mathrm{e}$, respectively ${ }^{24}$.

The transport sector demonstrates the largest divergence between top-down integrated assessment models and aggregation of bottom-up models. A key reason for this divergence is that place-based solutions and those that involve changing social norms and behavioural adaptations are hard to display in integrated assessment models (IAMs) ${ }^{26-29}$. A plethora of country- and city-specific solutions, many categorized into avoid and shift ( 15 and $18 \%$ of measures, respectively), is estimated to have the potential to bring GHG emissions in the transport sector down to $2.5 \mathrm{GtCO}_{2} \mathrm{e}^{30}$.

Key avoid strategies involve telecommuting, although total emission savings are estimated at no more than $1 \%$ of total land transport GHG emissions ${ }^{31}$. For example, COVID-19 confinement-induced telecommuting was compensated by more errands with cars, albeit recorded at shorter distances in California ${ }^{32}$. Urban planning, street space rededication, smart logistical systems and increased street connectivity with smaller distances have the largest potential to reduce the need for travel ${ }^{33,34}$, with a counterfactual potential of $25 \%$ reduction in urban energy use in 2050 considering only newly built cities (repercussion effects in the buildings sector are included in this estimate $)^{35}$. Improving transport nonetheless has the largest mitigation potential, in particular via electrification. In most ambitious transport energy models, full electrification of land transport and power-to-fuels for aviation and shipping can completely decarbonize the transport sector while also decreasing primary energy required per unit of end-use energy, in particular in electric land transport ${ }^{36}$. Vehicle lightweighting strategies can also lead to notable emissions savings through improved fuel economy ${ }^{37}$.

Avoiding energy use in buildings starts with adjusting dwelling size to household size, thus reducing overall demand for lighting and space conditioning. Smaller dwellings, shared space for housing and services and building lifespan extension all reduce the overall demand for carbon-intensive building materials such as concrete and steel ${ }^{38,39}$. It also includes designing buildings based 


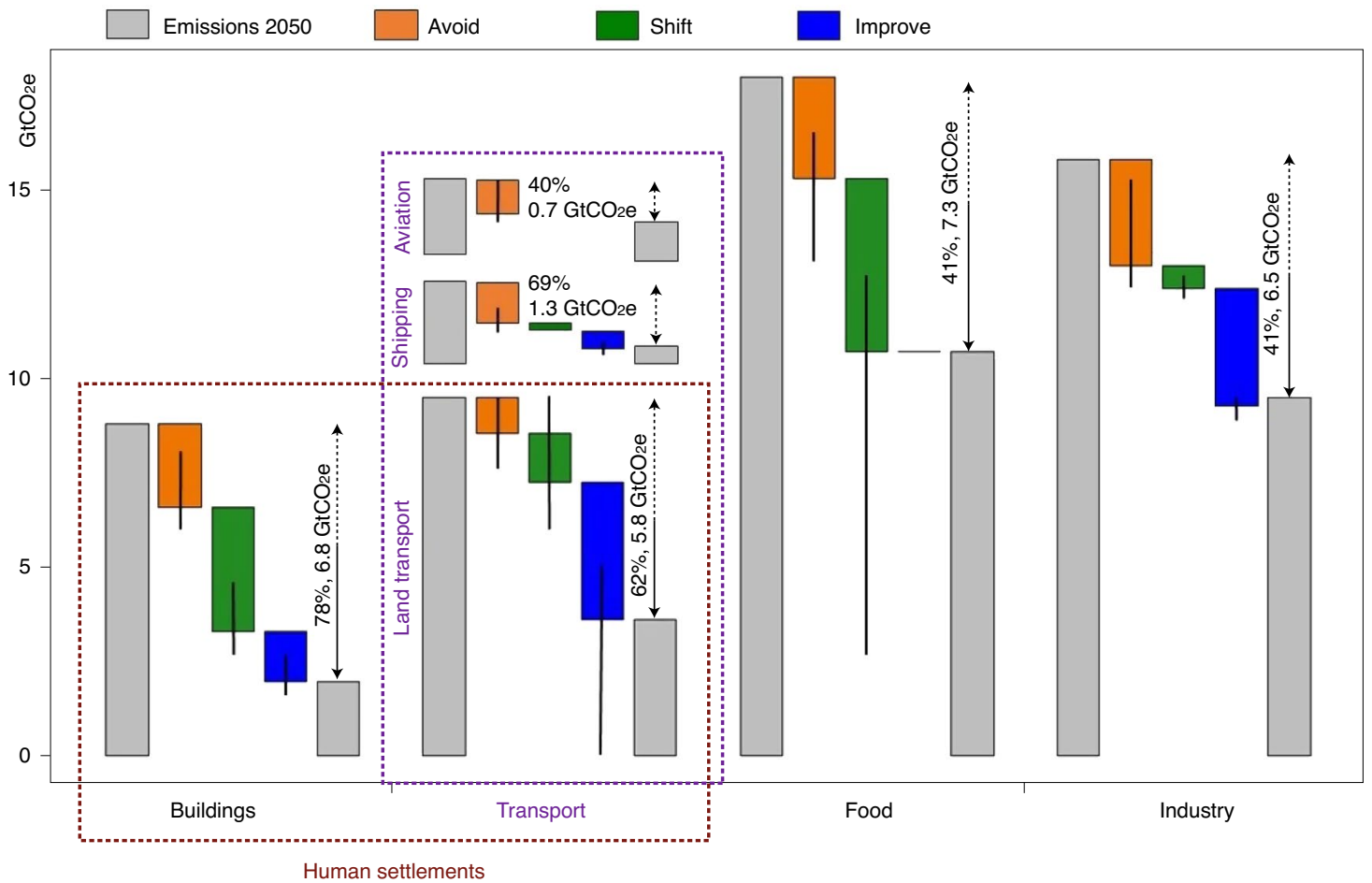

Fig. 1 | Mitigation potentials in end-use sector classified in ASI options. We reviewed studies estimating demand-side mitigation potentials associated with demand-side GHG ASI emission reduction strategies, and summarized the results as central values and full ranges (minimal to maximal potential, black error bars). Demand-side mitigation strategies technically could provide reductions of $78 \%\left(6.8 \mathrm{GtCO}_{2} \mathrm{e}\right), 62 \%\left(5.8 \mathrm{GtCO}_{2} \mathrm{e}\right), 41 \%\left(7.3 \mathrm{GtCO}_{2} \mathrm{e}\right)$ and $41 \%\left(6.3 \mathrm{GtCO}_{2} \mathrm{e}\right)$ in the buildings, transport, food and industry sectors, respectively (black dotted arrows). Urban strategies are merged with buildings and land transport strategies and are presented under 'human settlements' (red-dashed-line box). Transport demand-side mitigation strategies and potentials are grouped as land transport, shipping and aviation (purple-dashed-line box). To be able to give an approximation for the full potential across sectors, we ignore interaction effects between the three categories. Mitigation potentials are estimated against 2050 values of the IEA's stated policy scenario $^{58}$ and baseline assumption from the IPCC's SRCCL for food. Data sources and explanations are given in Table 1 and Supplementary Table 1.

on bioclimatic principles to maximize energy demand reduction through nature and building typology (single- versus multi-family buildings), adapting the size of buildings to the size of households and redesigning both individual energy end use and building operations: replace artificial light with daylighting ${ }^{40,41}$ and use lighting sensors to avoid demand for lumens from artificial light. Other options include designing passive houses that use thermal mass and smart controllers to avoid demand for space-conditioning services ${ }^{42}$, and eliminating standby power to reduce energy wasted in appliances/ devices (this alone may reduce household energy use by $10 \%)^{43}$. Three-dimensional printing of buildings further reduces construction waste, optimizes the geometries and minimizes the materials content of structural elements ${ }^{44}$. Overall, 'avoid' potential in the buildings sector, reducing waste in superfluous floor space, heating and IT equipment and energy use, has been estimated at $10 \%$ and $30 \%$, and possibly up to $50 \%$ (ref. ${ }^{45}$ ). Improve options, such as energy-efficient appliances, insulation and prosumer renewables on rooftops, may similarly reduce GHG emissions, combined, by $50 \%$ $(30-70 \%)^{42,46,47}$.

While demand-side solutions will change lifestyles, individuals have few opportunities to induce and realize demand-side solutions by themselves. In all three ASI categories, infrastructures and choice architectures play a crucial role. Avoid measures require structural change in organization management (for example, working time models that enable teleworking ${ }^{48}$ ), spatial structure (mixed use and compact cities to increase accessibility with active modes ${ }^{49}$ ), choice architectures (making healthy plant-based meals or coworking in shared spaces the default choice) and incentives (taxing land to incentivize more efficient use of floor space). Similarly, shift solutions require the availability of new modes of service provisionfor example, by offering shared pooled mobility ${ }^{50}$ and high-quality, plant-based diets ${ }^{51,52}$ as defaults, and regulation that prohibits high-emitting (and otherwise harmful) practices such as intensive animal farming and fossil-fuel-based heating and instead promote low-carbon solutions-for example, via social marketing incentivizing reduced red meat consumption ${ }^{53}$. Finally, improve options similarly require policy interventions such as carbon pricing, banning inefficient heating systems, lightbulbs and cars with internal combustion engines and diesel motors and mandating market shares of efficient technologies, planning procedures and practices. Making the purchase and management of low-carbon technologies the default, also in public facilities, is another key choice architecture intervention to accelerate the adoption of improve options ${ }^{54}$.

\section{Implications for near-term mitigation pathways}

It is instructive to compare our bottom-up assessment of sector-wise mitigation potentials with the literature on demand-side scenarios. The benchmark was provided by the low energy demand (LED) scenario modelled with the IAM MESSAGE ${ }^{55}$, with more recent scenarios emphasizing further opportunities by constraining GHG emissions further in the Global North ${ }^{56,57}$. A key difference is that LED focused on energy demand whereas the current assessment centres on GHG emissions as a mitigation metric. Energy demand is appropriate for demand-side evaluation because it separates carbon-intensity effects from supply-side measures, also enabling a clearer view of service-provisioning systems with low energy demand. GHG emission metrics have the advantage that non-energy sectors, and in particular, food for nutritional 
Table 1 | Demand-side mitigation strategies and mitigation potentials in end-use sectors

End-use sector $\mathrm{GtCO}_{2} \mathrm{e}$ in 2050 Mitigation strategy

\section{Range of $\mathrm{CO}_{2} \mathrm{e}$ References \\ emissions \\ reduction \\ potentials for \\ ASI}

Housing, leisure
and services
(buildings)
(total mitigation
potential: $78 \%$,

$6.8 \mathrm{GtCO}_{2} \mathrm{e}$ )

\section{8}

and services

(total mitigation

Mobility, accessibility (land transport) (total mitigation potential: $62 \%$, $5.8 \mathrm{GtCO}_{2} \mathrm{e}$ )
Nutrition (food) $\quad 16-20$ (central (total mitigation value: 18 ) potential: $41 \%$, (includes $7.3 \mathrm{GtCO}_{2} \mathrm{e}$ ) deforestation and land-use change emissions)
Avoid: sufficiency of energy and resources (include compact-city- and nature-based solutions from urban sector). Building design, size and use (behavioural and lifestyle change).

Shift: improve access and switch to renewables. On-site renewables, micro-grids, switch to lower-carbon fuels and electrification for space heating, cooling, cooking, hot water and electrical uses.

Improve: efficiency. Improved building envelope, improved building technical systems (for heating, ventilation, air conditioning, cooking and electrical uses), smart home and digitalization, efficient appliances, control systems, clean cooking.

9.5 (includes GHG Avoid: active travel in highly accessible cities; teleworking emissions from electrification of land transport) supported by compact, highly accessible city design and sa infrastructures for pedestrians and cyclists. Teleworking or telecommuters partially or entirely replace their out-of-home work activities by working at home or at locations close to home.

Shift: shared mobility and convenient and safe public transit. Pooled shared mobility with high occupancy and micro-mobility with high lifetime of vehicle stock; convenient rail-based public transit; supported by urban design and transit-oriented development, resulting in reduced travel distances; logistic optimization in last-mile freight.

Improve: BEVs, when charged with electricity generated from medium-decarbonized power system (IEA stated policies); behaviour change programmes on the socio-economic structures that impede adoption of BEVs; urban structures that enable reduced car dependence and how BEVs can assist grids; and synergies between emerging technologies and shared economy to maximizing the greater benefit of BEVs.

Avoid: food waste (overconsumption not further considered, as diets rich in calories-and, in particular, sugar-add little to GHG emissions).

Shift: animal-free protein. Switch to animal-free protein sources such as soy, lentils, other pulses and meat substitute products.

Products and

materials

(industry) (total

mitigation

potential: $41 \%$, $6.5 \mathrm{GtCO}_{2} \mathrm{e}$ )
Avoid: materials-efficient services. Avoid materials via dematerialization, the sharing economy, material-efficient and lightweight designs and yield improvements in manufacturing.

Avoid: lifespan extension. Designing products so that their lifetime can be extended through repair, refurbishing and remanufacturing, instigated via standardization, modularity and functional segregation.

Shift: reuse and recycling. Increasing the reusability and recyclability of product components. Example: dismantle old cars and reuse components for repairing other cars.

Improve: energy efficiency. Reduce the need for energy consumption through the installation of new, efficient technologies and through systems and operating practices that contribute to reduce energy needs.
10-40\% IEA 58 Kuhnhenn

(central value, et al ${ }^{79}$; Niamir et al. ${ }^{80}$; Ahl

25\%) et al. ${ }^{81}$; IGES et al. ${ }^{82}$; ECF ${ }^{83}$; Virage-énergie ${ }^{84}$

30-70\% IEA ${ }^{58}$; Niamir et al. ${ }^{85}$;

(central value, Mastrucci \& Rao ${ }^{86}$; IGES

$50 \%) \quad$ et al. ${ }^{82}$; ECF ${ }^{83}$; Mata et al. ${ }^{87}$; Virage-énergie ${ }^{84}$

30-65\% IEA ${ }^{58}$; Mata et al.88; IGES

(central value, et al. ${ }^{82}$; Ellsworth-Krebs

40\%) et al. ${ }^{89} ;$ ECF $^{83}$;

Virage-énergie $^{84}$

0-25\% Pomponi et al. ${ }^{90}$; Brand et al..$^{91}$;

(central value, Creutzig et al. ${ }^{32,55}$; Ivanova

10\%) et al. ${ }^{92}$; Riggs ${ }^{32}$; Mrkajic et al. ${ }^{11}$; Senbel et al. ${ }^{93}$

0-25\% Sheppard et al. ${ }^{94}$; ITF $95-99$;

(central value, Creutzig et al. ${ }^{5}$ $15 \%)$

30-100\% Ehrenberger et al. ${ }^{100}$; Hou (central value, et al. ${ }^{101}$; EEA ${ }^{102}$; Hill et al..$^{103}$; 50\%) Plötz et al. ${ }^{104}$; Khalili et al. ${ }^{36}$

8-25\% Clark et al. ${ }^{105}$; Makov et al. ${ }^{106}$; (central value, Poore \& Nemecek ${ }^{107}$; Schanes 15\%) et al. ${ }^{21}$; Gunders \& Bloom ${ }^{22}$; IPCC SRCCL 24; Hiç et al.108; Bajželj et al. ${ }^{2}$

$18-87 \%$ Clark et al. ${ }^{105} ;$ Semba et al. ${ }^{109}$; (central value, Springmann et al. ${ }^{110}$; Willett 40\%) (applies et al..$^{111}$; Parodi et al.112; IPCC to farm-gate SRCCL ${ }^{24}$; Bajželj et al. ${ }^{2}$ GHG emissions)

5-22\% IRP 113 ; Pauliuk et al. ${ }^{114}$; IEA ${ }^{58,115}$;

(central value, Grubler et al. ${ }^{55}$; Allwood \& 13\%) Cullen ${ }^{116}$; Carruth et al. ${ }^{17}$

3-7\% (central Lausselet et al.118; IEA ${ }^{58,115}$; value, 5\%) Cooper et al. ${ }^{119}$

4-7\% (central IEA ${ }^{58,115}$; Ellen MacArthur value, $5 \%$ ) Foundation ${ }^{120}$; IEA ${ }^{121}$; Material Economics ${ }^{122} ;$ Hertwich et al. ${ }^{38}$ 25-28\% Crijns-Graus et al. ${ }^{123}$; IEA ${ }^{58,115}$; (central value, Material Economics ${ }^{122}$ 25\%) 
Table 1 | Demand-side mitigation strategies and mitigation potentials in end-use sectors (continued)

\begin{tabular}{|c|c|c|c|c|}
\hline End-use sector & $\mathrm{GtCO}_{2} \mathrm{e}$ in 2050 & Mitigation strategy & $\begin{array}{l}\text { Range of } \mathrm{CO}_{2} \mathrm{e} \\
\text { emissions } \\
\text { reduction } \\
\text { potentials for } \\
\text { ASI }\end{array}$ & References \\
\hline $\begin{array}{l}\text { Mobility, } \\
\text { accessibility } \\
\text { (aviation) (total } \\
\text { mitigation } \\
\text { potential: } 40 \% \text {, } \\
0.7 \mathrm{GtCO}_{2} \mathrm{e} \text { ) }\end{array}$ & 1.8 & $\begin{array}{l}\text { Avoid: flights. Aviation is of low economic value and demand } \\
\text { is highly sensitive to prices. A carbon price of aviation fuel of } \\
\text { US } \$ 400 \mathrm{tCO}_{2}^{-1} \text { would halve demand for aviation in } 2050 \text {. }\end{array}$ & $\begin{array}{l}0-47 \% \\
\text { (central value, } \\
40 \%)\end{array}$ & $\begin{array}{l}\text { IATA }^{124} \text {; Schäfer et al. }{ }^{125} \\
\text { Sharmina et al. }\end{array}$ \\
\hline \multirow{3}{*}{$\begin{array}{l}\text { Mobility, } \\
\text { accessibility } \\
\text { (shipping) } \\
\text { (total mitigation } \\
\text { potential: } 69 \% \text {, } \\
1.3 \mathrm{GtCO}_{2} \mathrm{e} \text { ) }\end{array}$} & \multirow[t]{3}{*}{1.9} & $\begin{array}{l}\text { Avoid: reduce demand and slow steaming. Shifting supply } \\
\text { chains, lower demand for consumption goods and slow steaming } \\
\text { of ships would reduce shipping demand substantially. }\end{array}$ & $\begin{array}{l}40-60 \% \\
\text { (central value, } \\
47 \%)\end{array}$ & $\begin{array}{l}\text { Bouman et al. }{ }^{127} \text {, McKinnon }{ }^{128} \text {, } \\
\text { ITFF }^{129}\end{array}$ \\
\hline & & $\begin{array}{l}\text { Shift: modal shift to train. Shift from ships to long-distance } \\
\text { train (especially across the Eurasian continent) reduces GHG } \\
\text { emissions, but not }>1 \% \text { of expected emissions. }\end{array}$ & $\begin{array}{l}0-1 \% \text { (central } \\
\text { value, } 1 \%)\end{array}$ & $\operatorname{ITF}^{129}$ \\
\hline & & $\begin{array}{l}\text { Improve: design and power system. Independent of fuels (supply), } \\
\text { better hull design and improved propulsion system can make } \\
\text { ships much more efficient. }\end{array}$ & $\begin{array}{l}30-50 \% \\
\text { (central value, } \\
40 \%)\end{array}$ & $\begin{array}{l}\text { Bouman et al. }{ }^{127}, \text { McKinnon }^{128} \text {, } \\
\text { ITFF }^{129}\end{array}$ \\
\hline
\end{tabular}

Mitigation potential is estimated from sector-specific studies and models, and is reported in percentages to account for potentially diverging baselines and interaction terms with other mitigation strategies (valid if factorial decomposition between mitigation strategies is possible). Ranges reflect variability across assessments of the underlying literature. Baseline estimates for 2050 are from IEA stated policy scenario in energy sectors, and from the IPCC Special Report on Climate Change and Land (SRCCL) and additional sources in the food sector, and thus assume the absence of supply-side climate change mitigation (Supplementary information).

service, can also be evaluated on an equal basis. Because of different metrics and boundary conditions, a direct comparison between this assessment and LED is impossible. However, relative changes between energy/GHG emissions from 2020 until 2050 can be tentatively compared. In buildings, our assessment indicates a potential of up to $81 \%$ reduction between 2020 and 2050 whereas the LED scenario suggests $74-79 \%$ reduction in energy use for thermal comfort. One difference for our more optimistic value is the inclusion of prosumer-centric renewable energies as a demand-side measure. In transport, our assessment suggests up to $70 \%$ reduction between 2020 and 2050, which is higher than the 59-60\% change in energy demand in the LED scenario. A main reason for this difference is the accelerating uptake of electric vehicles by consumers, making a full-scale transition to electric vehicles by 2050 look possible. In industry, assessments produce similar values (21\% here, and $23 \%$ reduction in LED). Considering GHG emissions, we also assess the food sector, finding nearly $50 \%$ reduction potential by 2050 . These findings suggest that the LED scenario is plausible. Inversely it poses the question of why most other scenarios fail to consider these options. Reasons include the insufficient consideration of granular end-use technologies and the absence of representation of structural shifts in service-provisioning systems. In essence, high-level climate stabilization models have high resolution on the supply side but little exploration on the demand side.

Instructively, we compare our potential estimates to the International Energy Agency (IEA)'s Sustainable Development Scenario (SDS) for 2050 (ref. ${ }^{58}$ ). In three sectors, demand-side options can go a long way to reaching SDS levels, requiring only additional $1.2 \mathrm{GtCO}_{2}$ abatement on the supply side in buildings, $0.4 \mathrm{GtCO}_{2}$ in aviation and $0.1 \mathrm{GtCO}_{2}$ in shipping. In contrast, other sectors require more additional supply-side and land-use sector efforts to reach SDS levels: $10.5 \mathrm{GtCO}_{2}$ in food and land use, $5.5 \mathrm{GtCO}_{2}$ in industry and $3.2 \mathrm{GtCO}_{2}$ in land transport.

\section{Demand-side mitigation strategies improve well-being}

The IPCC's Special Report on Global Warming of $1.5^{\circ} \mathrm{C}$ provided evidence that energy demand solutions have more synergies and fewer trade-offs with sustainable development goals than energy supply-side solutions ${ }^{4}$ (Methods). Our own analysis of the weighted statistics shows that energy demand solutions have a ratio of synergies versus trade-offs with sustainable development goals (SDGs) of 5.3 (with most beneficial strategies in industry and buildings), whereas energy supply solutions show a ratio of 1.9 between synergies and trade-offs (Fig. 2). Demand-side solutions appear, hence, to be more beneficial to SDGs than supply-side solutions. While SDGs overlap with well-being, a detailed analysis of demand-side options on dimensions of well-being is missing.

Based on a database of 54,000 peer-reviewed articles and 604 with identified relevant input (Supplementary Tables 3-7), we analysed how sectoral demand-side and service-oriented mitigation strategies influence the constituents of well-being. We systematically coded whether mitigation strategies for each sector have positive, neutral or negative impact on the 18 constituents of well-being introduced in Fig. 3. We performed expert judgement by a team of between two and four researchers for each sector, also comprising explicit expertise on social sciences and well-being, and internal review by at least two other researchers, to code impact in categories on a scale of -3 to +3 , and substantiated this with evidence from the literature (Fig. 3). Confidence in these judgements varied, because both scale and multitude of effects vary across the underlying literature. In other cases, literature was missing even when the experts assumed relevant effects. Hence, we also provide confidence values associated with each mitigation-strategy/well-being-constituent couple (Extended Data Fig. 2) and report the confidence values together with the results of the well-being evaluation (Methods). The full table, including levels of agreement and evidence and literature substantiation of each entry, is provided in the Supplementary information.

Demand-side mitigation strategies have positive impacts on human well-being (high confidence). Our study shows that, among all demand-side option effects on well-being, 79\% (242 out of 306) are positive, $18 \%$ (56 out of 306) are neutral (or not relevant/specify) and $3 \%$ (8 out of 306) are negative. Active mobility (cycling and walking), efficient buildings and prosumer choices of renewable technologies have the most encompassing beneficial effects on well-being with no negative outcome detected. Urban and industry strategies are highly positive overall for well-being, but they will also 


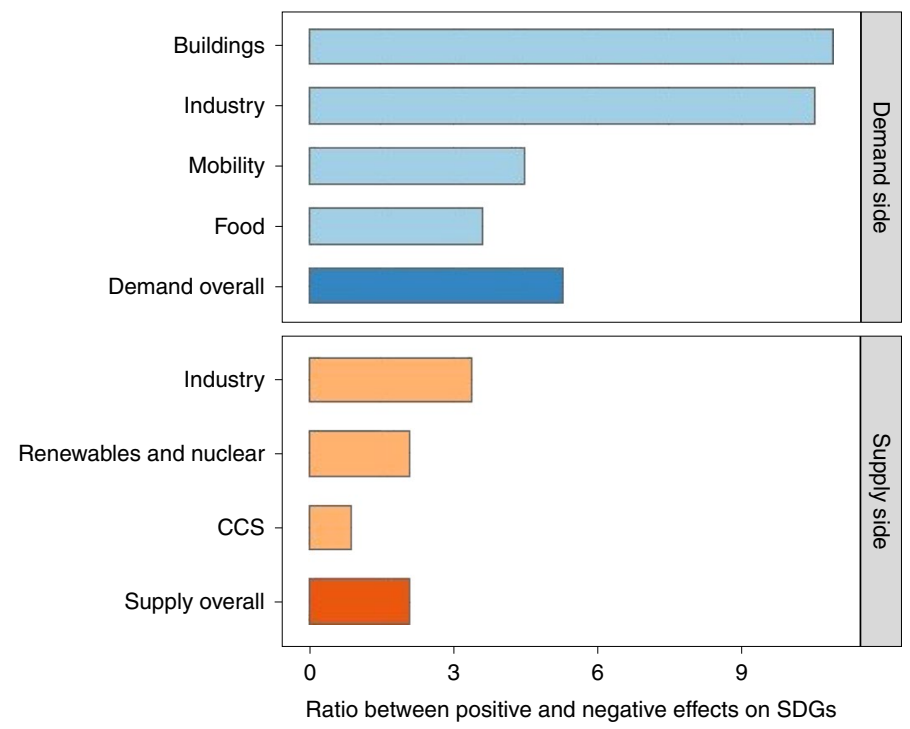

Fig. 2 | Ratio of weighted sum of synergies between SDGs and energy demand/supply solutions, and weighted sum of trade-offs between SDGs and demand/supply solutions. Sums were weighted according to confidence, as reported in ref. ${ }^{130}$ (that is, confidence with one star was weighted by 1 , confidence with two stars by 2, and so on). Ratios are similar for unweighted sums. CCS, carbon capture and storage.

\begin{tabular}{|c|c|c|c|c|c|c|c|c|c|c|c|c|c|c|c|c|c|c|}
\hline \multirow[b]{3}{*}{ Sectors } & SDGs & 2 & 6 & 7,11 & 3 & 6 & 7 & 11 & 11 & 4 & & \multicolumn{2}{|c|}{$11,2,8,10 \mid 5,10,16$} & \multirow{3}{*}{ 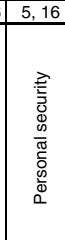 } & \multirow{3}{*}{ 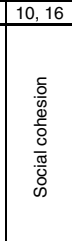 } & \multirow{3}{*}{ 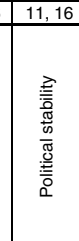 } & \multirow{3}{*}{ 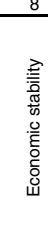 } & \multirow{3}{*}{ 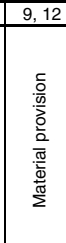 } \\
\hline & \begin{tabular}{|l} 
Mitigation strategies/well-being dimensions \\
\end{tabular} & \multirow[b]{2}{*}{8} & \multirow[b]{2}{*}{ 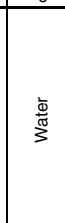 } & \multirow[b]{2}{*}{ 这 } & \multirow[b]{2}{*}{ 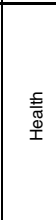 } & \multirow[b]{2}{*}{ 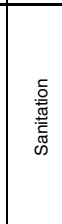 } & \multirow[b]{2}{*}{$\begin{array}{l}\text { 㧸 } \\
\text { 离 }\end{array}$} & \multirow[b]{2}{*}{$\begin{array}{l}\frac{\overline{\frac{\omega}{\omega}}}{\bar{\omega}} \\
\frac{\omega}{\omega}\end{array}$} & \multirow[b]{2}{*}{$\begin{array}{l}\text { 言 } \\
\stackrel{\overline{0}}{2}\end{array}$} & \multirow[b]{2}{*}{ 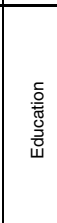 } & \multirow[b]{2}{*}{ 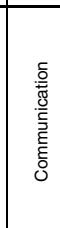 } & & & & & & & \\
\hline & 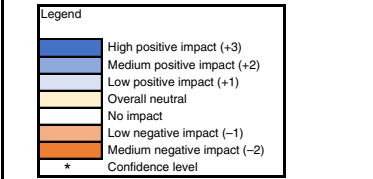 & & & & & & & & & & & 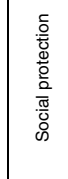 & 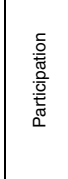 & & & & & \\
\hline \multirow{3}{*}{ 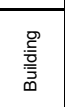 } & Sufficiency & $\underbrace{(+1)}_{\star \star * \star}$ & 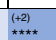 & 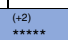 & $\frac{(+3)}{* * * * *}$ & $\stackrel{(+1)}{*}$ & $\frac{1+3)}{* * * *}$ & $\stackrel{(+1)}{*}$ & 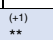 & 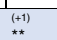 & $\frac{(+2)}{* \star *}$ & $\stackrel{\substack{(+1) \\
\star \star}}{x}$ & 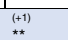 & & $\frac{(+2)}{*+2)}$ & & $\begin{array}{ll}(+2) \\
* * * *\end{array}$ & 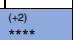 \\
\hline & Efficiency & $\left.\right|_{*} ^{+12)}$ & 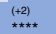 & 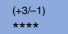 & 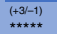 & $\stackrel{(+1)}{*}$ & 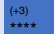 & 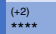 & & $\stackrel{(+1)}{* \pm \star}$ & $\stackrel{(+1)}{* \pm \neq}$ & & 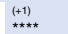 & 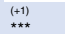 & 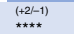 & & 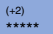 & 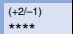 \\
\hline & Lower carbon and renewable energy & $(+2-1)$ & 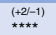 & $(:-3)+*$ & 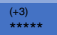 & & $(+3)$ & 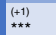 & (t+1) & $\stackrel{(+1)}{* \pm}$ & $\stackrel{(+2)}{* * *}$ & & $\stackrel{(+1)}{+1 *}$ & 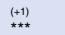 & 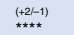 & & 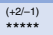 & 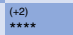 \\
\hline \multirow{3}{*}{ : } & Food waste & $\underbrace{(+1)}_{* \pm \neq}$ & $(+2)$ & $(+2)$, & $(+2)$ & $\stackrel{(1)}{* 1}$ & $\underset{\substack{(+1) \\
\star \star \star \star \star}}{(1)}$ & & & & 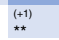 & $\left(\begin{array}{lll}(-1+1) \\
\star \star \star *\end{array}\right.$ & $\stackrel{(+1)}{* \star \star}$ & & & $\stackrel{(+1)}{*}$ & $(+1)$ & \\
\hline & Overconsumption & $(+1)$ & $\stackrel{(+1-1-1)}{*}$ & $\stackrel{(+1 /-1)}{*}$ & $(+3)$ & & $\stackrel{(+1 /-1)}{*}$ & & & & & & $\frac{(+2)}{* * *+*}$ & & & $\stackrel{(+1)}{*}$ & & \\
\hline & Animal-free protein & 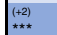 & 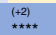 & 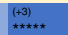 & $(+3)$ & & & & & & 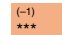 & 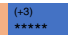 & 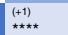 & & $(-1)$ & $(+2)$ & & \\
\hline \multirow{4}{*}{ 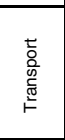 } & \begin{tabular}{|l} 
Teleworking and online education system \\
\end{tabular} & ${ }_{\substack{(+1) \\
\star \star}}$ & & 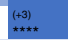 & $\underset{* \star 2)}{(+2)}$ & & $\begin{array}{l}(+2) \\
* * * * \\
* *\end{array}$ & $\stackrel{(+1)}{* *}$ & $\begin{array}{l}(+2) \\
* * * *\end{array}$ & 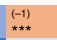 & $\stackrel{(+2)}{* *+*}$ & 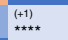 & $\begin{array}{l}(+2) \\
* * * *\end{array}$ & $\begin{array}{l}(+11-1) \\
* \star \star \star \star\end{array}$ & $\stackrel{(+2)}{*+*+*}$ & $\underset{(2)}{* * *}$ & $\stackrel{(+2)}{* *}$ & \\
\hline & Non-motorized transport & 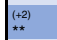 & $\stackrel{(+1)}{* *}$ & 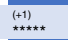 & $\begin{array}{l}(+3) \\
t+x+*\end{array}$ & & $\begin{array}{l}(+2) \\
* \star * \star *\end{array}$ & & ${ }_{* * * * * *}^{(+3)}$ & $l_{* *+1)}^{(+1)}$ & $\underset{* *+}{(4+3)}$ & 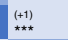 & $\stackrel{(+1)}{* \star}$ & $\begin{array}{l}(+2) \\
* * x+*\end{array}$ & $\stackrel{(+2)}{* 2 *}$ & $\stackrel{(+2)}{* *}$ & $\stackrel{(+2)}{* *}$ & \\
\hline & Shared mobility & $\underset{\substack{(+1) \\
* \star}}{*}$ & & $\begin{array}{l}(+3) \\
* * * \\
* *+\end{array}$ & $\left(\begin{array}{l}(+2) \\
* * * *\end{array}\right.$ & & $\begin{array}{l}(+1) \\
* \star \star \\
* \star\end{array}$ & & $\underset{(+2)}{* * \star *}$ & & 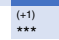 & $\begin{array}{l}(+2) \\
* * \star\end{array}$ & 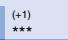 & $\underset{(1+1)-1)}{* \star \star}$ & 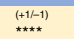 & $(-1)$ & 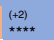 & 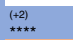 \\
\hline & BEVs & $\substack{(+1) \\
\star \star \star \star}_{(1)}$ & & 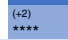 & 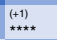 & $\underset{* t+1)}{(+1 *+}$ & $\begin{array}{l}1+3 \\
* * * *\end{array}$ & & $\begin{array}{l}(+2) \\
* * \star *\end{array}$ & & & $(+3)+\ldots$ & $\begin{array}{l}(+2) \\
\star \star \star \star\end{array}$ & & & & $\underset{1+2)}{* * * *}$ & $\stackrel{(-1)}{* *}$ \\
\hline & Compact city & $\underbrace{}_{\substack{(+2<-1) \\
* \star *}}$ & $\stackrel{(+1)}{*}$ & 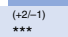 & 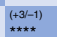 & $\stackrel{(+1)}{*}$ & 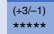 & 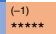 & $(+3)+* *$ & $(+1), \ldots$ & 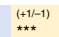 & $\left(\begin{array}{l}42) \\
* *\end{array}\right.$ & $\stackrel{(+1)}{* \pm}$ & $(+1)$ & 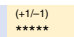 & & 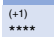 & $\stackrel{(+1)}{*}$ \\
\hline 듀 & Circular and shared economy & $\begin{array}{c}++2) \\
+* * *\end{array}$ & $\stackrel{(+1)}{* \star *}$ & $\begin{array}{l}(+2) \\
* \star *\end{array}$ & $\underset{\substack{(+2) \\
\star \star \star \star}}{*}$ & & $\begin{array}{l}(+3) \\
* * *\end{array}$ & $\begin{array}{l}(+2 l-1) \\
* \star \star\end{array}$ & ${ }_{* * * * *}^{(+3)}$ & $\underset{\substack{(+1) \\
* \star * *}}{(1)}$ & $\underset{\substack{(+1) \\
* \star \star \star}}{2}$ & 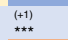 & $\underset{\substack{(+1) \\
* \star \star}}{*}$ & $\begin{array}{l}(+2) \\
* * * *\end{array}$ & 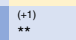 & $\underset{\substack{(+1) \\
* \star}}{4}$ & $\stackrel{1+2)}{* 2}$ & $\left(\begin{array}{lll}(-3) \\
* *\end{array}\right.$ \\
\hline 气ू & Systems approach in urban policy and practice & $\begin{array}{l}(+1) \\
\star \star \star\end{array}$ & $\begin{array}{l}(+2) \\
* \star *\end{array}$ & $\begin{array}{c}(+2) \\
\star \star \star x\end{array}$ & $\begin{array}{l}(+3) \\
* \star *\end{array}$ & $\underset{* \star 1)}{(+1)}$ & $\begin{array}{l}(+3) \\
* * \star \\
* *\end{array}$ & $\begin{array}{l}(+2) \\
\star \star \star *\end{array}$ & $\underset{* 3 *}{(+3)}$ & & $\stackrel{(+1)}{* *}$ & 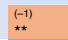 & $\stackrel{(+1)}{* \star \star}$ & $*+2)$ & $\stackrel{(+1)}{*}$ & & $\stackrel{(+1)}{*}$ & 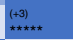 \\
\hline & Nature-based solutions & $\underset{\substack{(+2) \\
\star \star \star \star}}{(1)}$ & 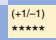 & $\begin{array}{l}(+3-1-1) \\
* * * *\end{array}$ & 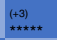 & $\underset{* 11)}{(+1)}$ & $\begin{array}{l}(+3) \\
* * *\end{array}$ & $\begin{array}{l}(+11-1) \\
\star \star \star\end{array}$ & $\stackrel{(+1)}{* \star *}$ & $\begin{array}{l}(+2) \\
* * * *\end{array}$ & & $\stackrel{(+2)}{* \star}$ & $\begin{array}{l}(+3) \\
* *\end{array}$ & $\underset{* \star \star}{(+1)}$ & $\underset{(1+2-2)}{* \star \star x}$ & & $\frac{1+3)}{* \cdots *}$ & $\stackrel{(+1)}{* *}$ \\
\hline & Using less material by design & $(+2)$ & $\begin{array}{l}(+2) \\
\star * \star\end{array}$ & $(: 3)$ & $\underset{(+2)}{* *}$ & $\underset{(1+2)}{* \star *}$ & $\underset{+3 * *}{(+3)}+$ & $\begin{array}{l}(+2) \\
* * * *\end{array}$ & $\stackrel{(+2)}{* * \star *}$ & $\stackrel{(+1)}{* \star}$ & $\begin{array}{l}(1+2) \\
* * *\end{array}$ & $\underset{* \star}{(+1)}$ & $\underset{\substack{(+1) \\
\star \star \star}}{2}$ & $\stackrel{(+1)}{* *}$ & $\stackrel{(+1)}{* *}$ & $\stackrel{(+1)}{* \star}$ & $\underset{\substack{(+2) \\
\star \star *}}{*}$ & $\begin{array}{l}(+3) \\
* *\end{array}$ \\
\hline$\underset{t}{\mathbb{E}}$ & Product life extension & $\mid \begin{array}{l}+22) \\
* *\end{array}$ & $\begin{array}{l}(+2) \\
\star \star \star *\end{array}$ & 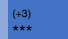 & $(+2)$ & $\stackrel{(+2)}{* \star 2}$ & $\begin{array}{l}(+3) \\
* * * *\end{array}$ & $\stackrel{(+2)}{* \star * *}$ & $\underset{\substack{(+2) \\
* \star \star \star *}}{2}$ & $\underset{*+1)}{(+1)}$ & $\stackrel{(+2)}{* 2 \times}$ & $\underset{\substack{(+1) \\
* *}}{n}$ & $\begin{array}{l}(-1) \\
* * *+\end{array}$ & $\underset{* \star}{(+1)}$ & $\underset{\substack{(+1) \\
\star \star}}{(2)}$ & $\stackrel{(+1)}{* \star}$ & $\begin{array}{l}(+2) \\
\star \star \star \star\end{array}$ & $(+3)$ \\
\hline$\underline{\underline{z}}$ & Energy efficiency & $\mid \begin{array}{ll}+22) \\
* *\end{array}$ & $\stackrel{(+2)}{* \star \star}$ & $\begin{array}{l}(+3)) \\
* * *\end{array}$ & $\stackrel{(+1)}{* \pm}$ & $\begin{array}{l}(+2) \\
\star \star \star\end{array}$ & $\begin{array}{l}(+3) \\
* * * *\end{array}$ & $\stackrel{(+2)}{* 2 \times}$ & $\stackrel{(+2)}{* \star \star * *}$ & $\underset{(+1)}{(x \star)}$ & $\begin{array}{l}(+2) \\
* *\end{array}$ & $\begin{array}{l}(+2) \\
* * *+*\end{array}$ & $\stackrel{(+2)}{* \star 2}$ & $\underset{\star(+1)}{(+1)}$ & & $\stackrel{(+1)}{\star \star}$ & $\underset{(+2)}{* \star *}$ & $\stackrel{(+2)}{* \pm}$ \\
\hline & Circular economy & $\underset{\substack{1+22) \\
* \star *}}{2}$ & 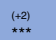 & $\begin{array}{l}(+3) \\
* * *\end{array}$ & $\begin{array}{l}(+1) \\
* \star\end{array}$ & $\begin{array}{l}(+2) \\
* \star *\end{array}$ & $\begin{array}{l}(+3) \\
* * * *\end{array}$ & $\begin{array}{l}(+2) \\
* * * *\end{array}$ & $\underset{\substack{(+2) \\
* \star * \star *}}{2}$ & ${ }_{*+1}^{(+1)}$ & $\begin{array}{l}(+2) \\
* * *\end{array}$ & $\underset{(+1)}{(+1)}$ & $\underset{\substack{(+1) \\
* \star \star}}{(2)}$ & $\begin{array}{l}(+2) \\
* * \\
* *\end{array}$ & $\underset{\substack{(+1) \\
\star \star}}{(1)}$ & & $\begin{array}{l}(+2) \\
* \star *\end{array}$ & $\left(\begin{array}{ll}(3) \\
* *\end{array}\right.$ \\
\hline
\end{tabular}

Fig. 3 | Effects of demand-side options on well-being in 19 different categories. Magnitude and direction of the well-being effect. Detailed data underpinning the assessment are reported in Supplementary Tables 3-7.

reshape supply-side businesses with transient intermediate negative effects. Shared mobility, like all others, has overall highly beneficial effects on well-being but also displays a few negative consequences, depending on implementation, such as a minor decrease in personal security for patrons of ridesourcing (connecting drivers with passengers via apps operated by platform providers). Differentiation, however, is important. For example, shared pooled mobility provides more urban benefits, and also higher climate change mitigation potential, as compared to ridesourcing ${ }^{50}$.

Positive links between mitigation measures and well-being are 19 fold greater than negative links. Although confidence in 50\% of all cases is medium to high (between 3 and 5 on a scale of $0-5$ ), it is higher in the physical constituents and comparatively low in the social constituents of well-being.

The highest benefits are observed in air, health and energy (high confidence levels), food (medium confidence), mobility (high confidence), economic stability (high confidence) and water (medium-high confidence). Although the relation between demand-side mitigation strategies and the social aspects of human well-being is important, this is less reflected in the literature to date and hence our assessment finds more neutral/unknown interactions. 


\section{Box 1 | Evaluation of well-being in demand-side climate change mitigation}

In economics, evaluations of well-being are predominantly based on the assumption that preference satisfaction constitutes welfare and that such preferences are given (unchanged by policy). On a simple interpretation of this normative position, demand for goods and services is, by definition, good for well-being. For sustainability transitions this is, from most perspectives in social science, an overly limited view of well-being ${ }^{131}$ because it ignores the fact that changes in preferences can be an integral part of societal transitions ${ }^{132}$. With fixed preferences, only changes in relative prices will reduce emissions, not changes in citizens' desires, environmental motives or social norms ${ }^{133}$. In these circumstances, straightforward assessment of policies by their costs is circular, and hence insufficient, because it ignores potential well-being outcomes resulting from collective shifts in preferences (Box Table 1).

For transitioning to a low-carbon society, several pieces of evidence indicate an important role for endogenous preferences: first, humans can absorb low-carbon food and other preferences and conventions (for example, seatbelts) through policy interventions ${ }^{134}$. Second, learning about route $^{135}$ and mode choice $^{136,137}$ have been documented as changing transport decisions. Third, peer effects exist for both car purchases ${ }^{138}$ and solar panel uptake ${ }^{139}$ : a policy that makes a low-carbon good more attractive can change preferences by influencing the social norm. Fourth, in an experimental setting, carbon pricing can also change preferences by crowding out citizens' intrinsic motivation to choose low-carbon products as documented ${ }^{140}$. For actual carbon tax reforms, however, carbon taxes make consumers reduce emissions more than could be expected from the relative price

Well-being improvements are most notable in health quality ( 0.61 on average across all mitigation options on a scale from -1 to $+1)$, air (0.59) and energy (0.57). These categories are also most substantiated in the literature, often under the framing of cobenefits. In many cases, cobenefits outweigh the mitigation benefits of specific GHG emission reduction strategies. This includes clean-cook stoves (for example, powered by liquified petroleum gas) that can improve the livelihood of $>40 \%$ of the world's population by reducing indoor air pollution ${ }^{59}$; it includes cobenefits from improved outdoor air quality in cities, resulting from reduced private motorized mobility using combustion and diesel engines, and from more active mobility ${ }^{60,61}$, often associated with the more accessible environments of compact cities $^{62}$; and it includes a shift away from high-emission diets that would improve public health considerably, especially in high-income countries ${ }^{63}$.

Food (0.50), mobility (0.46) and water (0.41) are further categories where well-being is improved. Mobility has entries with the highest well-being rankings for teleworking, compact cities and urban system approaches. Effects on well-being in water and sanitation mostly come from buildings and urban solutions.

Social dimensions, such as personal security, social cohesion and, especially, political stability, are less predominantly represented. An exception is economic stability (0.52), suggesting that demand-side options generate stable opportunities to participate in economic activities. Altogether, the literature on social constituents, in relation to climate change mitigation, is meagre. However, there are still clear indications that many demand-side mitigation strategies also have potential to improve the social constituents of well-being. For example, the predominant contribution of clean-cook stoves may relate to the well-being of women, who would require less time for biomass collection and cooking and could better participate in economic and social life ${ }^{64}$. Compact cities and urban system solutions
Box Table 1 | The assumption that preferences are exogenous or endogenous has an impact on the evaluation of solutions

\begin{tabular}{|c|c|c|}
\hline & $\begin{array}{l}\text { Supply-side } \\
\text { solutions }\end{array}$ & Demand-side solutions \\
\hline Exogenous preferences & $\begin{array}{l}\text { Current patterns } \\
\text { of service } \\
\text { provisions are } \\
\text { appropriate, and } \\
\text { new technologies } \\
\text { must substitute } \\
\text { current } \\
\text { supply-side } \\
\text { technologies } \\
\text { closely. }\end{array}$ & $\begin{array}{l}\text { Making existing } \\
\text { technologies more } \\
\text { efficient (improve) } \\
\text { is appropriate, but } \\
\text { shifting or reducing } \\
\text { consumption patterns are } \\
\text { insufficiently considered. } \\
\text { Social dynamics are } \\
\text { often directed to enable } \\
\text { overconsumption. }\end{array}$ \\
\hline Endogenous preferences & $\begin{array}{l}\text { Lack of orientation } \\
\text { on what should } \\
\text { be produced; } \\
\text { alternative } \\
\text { (partially } \\
\text { objective) metrics } \\
\text { are required. }\end{array}$ & $\begin{array}{l}\text { Societies can choose to } \\
\text { modify service-provisioning } \\
\text { systems and lifestyles; } \\
\text { alternative metrics and } \\
\text { institutions are required. }\end{array}$ \\
\hline
\end{tabular}

change alone ${ }^{141,142}$, consistent with a change in preferences. In cases of other transitions, such as in smoking, there is also evidence that societies guided the processes of shifting preferences and that societal values changed along with policies and relative prices ${ }^{143}$ (this is also true conversely: interest groups may shape preferences and values to hinder transitions ${ }^{144}$ ).

have strong, albeit ambiguous, effects on well-being, and positive outcomes depend on urban design ${ }^{65,66}$. Teleworking is ambiguous: if designed without face-to-face interaction, teleworking may result in social isolation ${ }^{67}$.

Much public attention is paid to fossil-fuel-intensive incumbents and the costs of transitioning, suggesting a macroeconomic manifestation of loss aversion. Our analysis shows that demand-side solutions indeed reduce the economic performance of incumbents, especially in the extractive industry (Fig. 3, last column). Sufficiency measures in buildings require fewer materials and appliances, animal-free protein compromises the economic outlook of the agricultural meat industry while active and shared mobility, together with more accessible, compact cities, implicate a smaller market for cars (Supplementary Tables 3-7). Importantly, economic stability as a whole is still assessed positively (compare the corresponding column in Fig. 3) -for example, because demand-side options also generate new jobs in new industries (Supplementary Tables 3-7).

A transition requires not only investments into new products and business models with an uncertain outlook on future profits, but also a psychological transition away from mental models and expertise that were successful in the past ${ }^{68}$. For example, many of Germany's engineers are proud of their world-class expertise in the manufacture of internal combustion engines, and acknowledging that this expertise now lacks a viable future is a considerable psychological challenge and a call for strategic planning ${ }^{69}$. Similarly, the meat industry is organized around efficient and highly scaled animal meat production, challenged by a transformation towards low-carbon-intensive nutrition services. The concerns of supply-side incumbents must hence be explicitly addressed-for example, by developing just transition opportunities for workers and employees.

Nearly all well-being effects depend on both individual and cultural preconditions (for example, the previous level of red meat 
consumption). Our well-being evaluation refers to mean expected effects, understanding that well-being effects can vary considerably with circumstances. Confidence is highest for the well-being dimensions air, health and mobility, and for the mitigation options compact city, non-motorized transport and building-level sufficiency. The well-being dimensions education, shelter and political stability have lowest confidence, reflecting a respective scarcity in the literature.

\section{Prioritizing demand-side options according to well-being}

Well-being analysis closes an epistemic gap, and the concurrent analysis of demand-side mitigation options and their well-being effects allows for tentative prioritization of mitigation options. Further quantification and ranking of mitigation options should nonetheless also include aggregate additional quantifications where possible and, in particular in the air quality and health domains, where effects have been well quantified and where high and notable effects have been demonstrated ${ }^{70,71}$.

A combined assessment of mitigation effect and well-being suggests that, in the food sector, shifting to plant-based diets is a main option in the developed world: it delivers potentially $40 \%$ or even higher reduction in GHG emissions while reducing global mortality by $6-10 \%$, equalling health cobenefits of $0.4-13 \%$ of global GDP ${ }^{63}$.

In the mobility sector the dominant mitigation option is a transition to electric mobility in land transport, enabling, in combination with a $100 \%$ renewable electricity sector, a reduction of GHG emission in land transport to zero before 2050 at the country level ${ }^{72}$. This strategy improves air quality and concurrent health and, for example, as part of an overall mitigation strategy the annualized monetary benefits outweigh the mitigation costs by a factor of two in the case of California ${ }^{70}$. However, a shift to active, non-motorized transport achieves even larger health benefits than electrification of transport ${ }^{60,73}$. While active mobility has less potential than battery electric vehicles (BEVs) for mitigation, its additional effect would not only further amplify health benefits but also alleviate the burden of very high BEV scale-up until 2050. Active mobility also aligns with dominant urban sector strategies and, in particular, compact cities that enable accessibility with non-motorized transport.

In the buildings sector, the design, size and use of the built environment, combined with a plethora of technology adoptions, can decrease emissions substantially until 2050, ranging from insulation to smart appliances to prosumer renewable energy provision. In that sector, although health and air quality effects are well quantified and notable ${ }^{74}$, visible solar photovoltaic panels and digital tools for energy saving are also related to belonging and self-efficacy ${ }^{75,76}$, thus contributing to the social domains of well-being.

Evaluation of well-being requires an understanding of malleable preferences, and the opportunity to redesign infrastructures and services in different ways (Box 1 and Supplementary Table 2). Behavioural responses, including dietary and mobility choices, depend on choices of new service delivery systems, new products, infrastructure designs and access to technologies. Infrastructures and technologies enable a shift to low- or zero-carbon lifestyles. This is important for pragmatic climate change mitigation policy design, emphasizing the importance of low-carbon infrastructure and service provisions. For example, in transport, planning decisions are often made based on observed demand of transport modes and distances. Instead, our evaluation suggests that a high quality of accessibility can be justified as a starting point for transport planning.

There are three limitations and associated directions for future research in co-evaluation of climate change mitigation and well-being. First, the social constituents of well-being deserve better quantification. Systematically advanced case studies, with quantification where possible and aggregate studies of demand-side mitigation options and their social dimensions at country level-for example, as captured by the World Value Survey-are important.
Second, empirical studies and mechanistic and causal modelling needs to much more effectively address the relationships between broad climate change mitigation options and the social constituents of well-being. For example, while the health benefits of active travel and reduced noise of motorized transport in cities are mostly understood, it is less clear how greater space availability for pedestrians translates into participation, trust and social protection. Differentiated insights from psychology, neuroscience and sociology deserve more attention in the joint assessment of climate change mitigation options and well-being. Third, future research should also assess potential interaction effects for ASI demand-side options. This could be done by IAMs if these find a comprehensive way to capture behavioural responses by end users. More modelling, with both IAMs and sector-specific general equilibrium models, is needed to represent accurately the behavioural effects involved in the cobenefits of mitigation actions. Smaller general equilibrium models, focusing on individual sectors, are also well suited to better assessment of the effects of multiple constituencies on well-being. For example, modelling captures the premise that, with food and transport choices, the low-carbon option makes people healthier because it incentives them to eat a healthier diet and exercise more $^{77,78}$.

\section{Climate mitigation and human well-being}

Our results matter regarding the core challenge of climate change mitigation. Even the most optimistic upscaling of low-carbon technologies would remain insufficient to meet currently projected energy demand in 2050, as approximately required by the Paris Agreement. Demand-side reduction strategies hence provide the essential breathing space needed for meeting climate targets in the short and medium term. We also show that these are consistent with improved well-being.

Further research at higher resolution levels on serviceprovisioning systems that reduce GHG emissions while maintaining or improving constituents of well-being will be highly policy relevant. This is particularly true for developing countries, as most of the options evaluated have been studied in developed countries. A new configuration of work and service-provisioning models consistent with low GHG emissions and resource demand is based on appropriate evaluation of well-being. Such a configuration needs to supplement cost-based macroeconomic metrics with consistent and direct measurements of the constituents of well-being, and to include access to health systems, shelter, high-quality nutrition and safe social environments. Our contribution makes an integral step to this evaluation by systematically assessing the literature on demand-side mitigation options through the lens of well-being. We demonstrate their large mitigation potential-starting with a perspective on what people need for a good life adds compelling options to the space of climate change mitigation solutions.

\section{Online content}

Any methods, additional references, Nature Research reporting summaries, source data, extended data, supplementary information, acknowledgements, peer review information; details of author contributions and competing interests; and statements of data and code availability are available at https://doi.org/10.1038/ s41558-021-01219-y.

Received: 13 December 2020; Accepted: 11 October 2021; Published online: 25 November 2021

\section{References}

1. Mundaca, L., Ürge-Vorsatz, D. \& Wilson, C. Demand-side approaches for limiting global warming to $1.5^{\circ} \mathrm{C}$. Energy Effic. 12, 343-362 (2019).

2. Bajželj, B. et al. Importance of food-demand management for climate mitigation. Nat. Clim. Change 4, 924-929 (2014). 
3. Creutzig, F. et al. Towards demand-side solutions for mitigating climate change. Nat. Clim. Change 8, 268-271 (2018).

4. IPCC Special Report on Global Warming of $1.5^{\circ} \mathrm{C}$ (eds Masson-Delmotte, V. et al.) (WMO, 2018).

5. Creutzig, F. et al. Beyond technology: demand-side solutions for climate change mitigation. Annu. Rev. Environ. Resour. 41, 173-198 (2016).

6. Deeming, C. Addressing the social determinants of subjective wellbeing: the latest challenge for social policy. J. Soc. Policy 42, 541-565 (2013).

7. Stiglitz, J., Sen, A. \& Fitoussi, J.-P. The Measurement of Economic Performance and Social Progress Revisited: Reflections and Overview (OFCE, 2009); https://www.researchgate.net/publication/278828759_The Measurement_of_Economic_Performance_and_Social_Progress_Revisited_ Reflections_and_Overview

8. Durand, M. The OECD better life initiative: how's life? and the measurement of well-being. Rev. Income Wealth 61, 4-17 (2015).

9. Fleurbaey, M. \& Blanchet, D. Beyond GDP: Measuring Welfare and Assessing Sustainability (Oxford Univ. Press, 2013).

10. Roger, C. Well-being in The Stanford Encyclopedia of Philosophy (ed. Zalta, E. N.) (The Metaphysics Research Lab, 2008); http://plato.stanford.edu/ archives/win2008/entries/well-being

11. Mrkajic, V., Vukelic, D. \& Mihajlov, A. Reduction of $\mathrm{CO}_{2}$ emission and non-environmental co-benefits of bicycle infrastructure provision: the case of the University of Novi Sad, Serbia. Renew. Sustain. Energy Rev. 49, 232-242 (2015).

12. Lamb, W. F. \& Steinberger, J. K. Human well-being and climate change mitigation. Wiley Interdiscip. Rev. Clim. Change 8, e485 (2017)

13. Mattauch, L., Ridgway, M. \& Creutzig, F. Happy or liberal? Making sense of behavior in transport policy design. Transp. Res. D Transp. Environ. 45, 64-83 (2015)

14. Sen, A. in The Quality of Life (eds Nussbaum, M. \& Sen, A.) Ch. 5 (Clarendon Press, 1993); https://scholar.harvard.edu/sen/publications/ capability-and-well-being-0

15. Max-Neef, M., Elizalde, A. \& Hopenhayn, M. in Real-Life Economics: Understanding Wealth Creation (eds Ekins, P. \& Max-Neef, M.) 197-213 (Routledge, 1992).

16. Dalkmann, H. \& Brannigan, C. Transport and Climate Change. Sustainable Transport: A Sourcebook for Policy-makers in Developing Cities (GTZ, 2007); https://lib.icimod.org/record/13155

17. Bongardt, D. et al. Low-Carbon Land Transport: Policy Handbook (Routledge, 2013).

18. van den Berg, N. J. et al. Improved modelling of lifestyle changes in integrated assessment models: cross-disciplinary insights from methodologies and theories. Energy Strategy Rev. 26, 100420 (2019).

19. Roy, J., Some, S., Das, N. \& Pathak, M. Demand side climate change mitigation actions and SDGs: literature review with systematic evidence search. Environ. Res. Lett. 16, 043003 (2021).

20. Food Wastage Footprint: Full-Cost Accounting (FAO, 2014).

21. Schanes, K., Dobernig, K. \& Gözet, B. Food waste matters-a systematic review of household food waste practices and their policy implications. J. Clean. Prod. 182, 978-991 (2018).

22. Gunders, D. \& Bloom, J. Wasted: How America is Losing up to 40 Percent of its Food from Farm to Fork to Landfill (NRDC, 2017); https://www.nrdc.org/ resources/wasted-how-america-losing-40-percent-its-food-farm-fork-landfill

23. Wilson, N. L., Rickard, B. J., Saputo, R. \& Ho, S.-T. Food waste: the role of date labels, package size, and product category. Food Qual. Prefer. 55, 35-44 (2017).

24. Shukla, P. R. et al. (eds) Special Report on Climate Change and Land (IPCC, 2019).

25. Smith, P. et al. in Climate Change 2014: Mitigation of Climate Change (eds Edenhofer, O. et al.) 811-922 (IPCC, Cambridge Univ. Press, 2014).

26. Creutzig, F. Evolving narratives of low-carbon futures in transportation. Transp. Rev. 36, 341-360 (2015).

27. McCollum, D. L. et al. Improving the behavioral realism of global integrated assessment models: an application to consumers' vehicle choices. Transp. Res. D Transp. Environ. 55, 322-342 (2017).

28. Geels, F. W., Sovacool, B. K., Schwanen, T. \& Sorrell, S. The socio-technical dynamics of low-carbon transitions. Joule 1, 463-479 (2017).

29. Larkin, A., Hoolohan, C. \& McLachlan, C. Embracing context and complexity to address environmental challenges in the water-energy-food nexus. Futures 123, 102612 (2020).

30. Gota, S., Huizenga, C., Peet, K., Medimorec, N. \& Bakker, S. Decarbonising transport to achieve Paris Agreement targets. Energy Effic. 12, 363-386 (2019).

31. Shabanpour, R., Golshani, N., Tayarani, M., Auld, J. \& Mohammadian, A. Analysis of telecommuting behavior and impacts on travel demand and the environment. Transp. Res. D Transp. Environ. 62, 563-576 (2018).

32. Riggs, W. Telework and sustainable travel during the COVID-19 era. Preprint at SSRN https://doi.org/10.2139/ssrn.3638885 (2020).

33. Policy Pathways: A Tale of Renewed Cities (International Energy Agency, 2013).
34. Creutzig, F. et al. Transport: a roadblock to climate change mitigation? Science 350, 911-912 (2015).

35. Creutzig, F., Baiocchi, G., Bierkandt, R., Pichler, P.-P. \& Seto, K. C. Global typology of urban energy use and potentials for an urbanization mitigation wedge. Proc. Natl Acad. Sci. USA 112, 6283-6288 (2015).

36. Khalili, S., Rantanen, E., Bogdanov, D. \& Breyer, C. Global transportation demand development with impacts on the energy demand and greenhouse gas emissions in a climate-constrained world. Energies 12, 3870 (2019).

37. IPCC Climate Change 2014: Mitigation of Climate Change (eds Edenhofer, O. et al.) (Cambridge Univ. Press, 2014).

38. Hertwich, E. G. et al. Material efficiency strategies to reducing greenhouse gas emissions associated with buildings, vehicles, and electronics-a review. Environ. Res. Lett. 14, 043004 (2019).

39. Pauliuk, S. et al. Global scenarios of resource and emissions savings from systemic material efficiency in buildings and cars. Nat. Commun. 12, 5097 (2021).

40. Belussi, L. et al. A review of performance of zero energy buildings and energy efficiency solutions. J. Build. Eng. 25, 100772 (2019).

41. Bodart, M. \& De Herde, A. Global energy savings in offices buildings by the use of daylighting. Energy Build. 34, 421-429 (2002).

42. Ürge-Vorsatz, D. et al. Advances toward a net-zero global building sector. Annu. Rev. Environ. Resour. 45, 227-269 (2020).

43. Roy, J., Dowd, A., Muller, A., Pal, S. \& Prata, N. in Global Energy Assessment-Toward a Sustainable Future (eds Global Energy Assessment Writing Team) 1527-1548 (Cambridge Univ. Press/The International Institute for Applied Systems Analysis, 2012).

44. Dixit, M. K. 3-D printing in building construction: a literature review of opportunities and challenges of reducing life cycle energy and carbon of buildings. IOP Conf. Ser. Earth Environ. Sci. 290, 012012 (2019).

45. Nadel, S. \& Ungar, L. Halfway There: Energy Efficiency Can Cut Energy Use and Greenhouse Gas Emissions in Half by 2050 (ACEEE, 2019); https://www. aceee.org/research-report/u1907

46. Nisa, C. F., Bélanger, J. J., Schumpe, B. M. \& Faller, D. G. Meta-analysis of randomised controlled trials testing behavioural interventions to promote household action on climate change. Nat. Commun. 10, 4545 (2019).

47. Wang, H., Chen, W. \& Shi, J. Low carbon transition of global building sector under 2- and 1.5-degree targets. Appl. Energy 222, 148-157 (2018).

48. Hook, A., Court, V., Sovacool, B. K. \& Sorrell, S. A systematic review of the energy and climate impacts of teleworking. Environ. Res. Lett. 15, 09003 (2020).

49. Ewing, R. \& Cervero, R. 'Does compact development make people drive less?' The answer is yes. J. Am. Plann. Assoc. 83, 19-25 (2017).

50. Creutzig, F. Making Smart Mobility Sustainable (Israel Public Policy Institute, 2020); https://www.ippi.org.il/smart-shared-mobility-experts workshop

51. Vecchio, R. \& Cavallo, C. Increasing healthy food choices through nudges: a systematic review. Food Qual. Prefer. 78, 103714 (2019).

52. Bauer, J. M., Bietz, S., Rauber, J. \& Reisch, L. A. Nudging healthier food choices in a cafeteria setting: a sequential multi-intervention field study. Appetite 160, 105106 (2021)

53. Bogueva, D., Marinova, D. \& Raphaely, T. Reducing meat consumption: the case for social marketing. Asia Pac. J. Mark. Logist. 29, 477-500 (2017).

54. Delgado, L. \& Shealy, T. Opportunities for greater energy efficiency in government facilities by aligning decision structures with advances in behavioral science. Renew. Sustain. Energy Rev. 82, 3952-3961 (2018).

55. Grubler, A. et al. A low energy demand scenario for meeting the $1.5^{\circ} \mathrm{C}$ target and sustainable development goals without negative emission technologies. Nat. Energy 3, 515-527 (2018).

56. Millward-Hopkins, J., Steinberger, J. K., Rao, N. D. \& Oswald, Y. Providing decent living with minimum energy: a global scenario. Glob. Environ. Change 65, 102168 (2020)

57. Keyßer, L. T. \& Lenzen, M. $1.5^{\circ} \mathrm{C}$ degrowth scenarios suggest the need for new mitigation pathways. Nat. Commun. 12, 2676 (2021).

58. World Energy Outlook 2020 (IEA, 2020); https://www.iea.org/reports/ world-energy-outlook-2020

59. Grieshop, A. P., Marshall, J. D. \& Kandlikar, M. Health and climate benefits of cookstove replacement options. Energy Policy 39, 7530-7542 (2011).

60. Woodcock, J. et al. Public health benefits of strategies to reduce greenhouse-gas emissions: urban land transport. Lancet 374, 1930-1943 (2009).

61. Creutzig, F., Mühlhoff, R. \& Römer, J. Decarbonizing urban transport in European cities: four cases show possibly high co-benefits. Environ. Res. Lett. 7, 044042 (2012)

62. Ahmad, S., Goodman, A., Creutzig, F., Woodcock, J. \& Tainio, M. A comparison of the health and environmental impacts of increasing urban density against increasing propensity to walk and cycle in Nashville, USA. Cities Health 4, 55-65 (2020).

63. Springmann, M. et al. Mitigation potential and global health impacts from emissions pricing of food commodities. Nat. Clim. Change 7, 69-74 (2017) 
64. Mazorra, J., Sánchez-Jacob, E., de la Sota, C., Fernández, L. \& Lumbreras, J. A comprehensive analysis of cooking solutions co-benefits at household level: healthy lives and well-being, gender and climate change. Sci. Total Environ. 707, 135968 (2020).

65. Burton, E. in Sustainable Urban Form (eds Burton, E. et al.) 19-29 (Routledge, 2000).

66. Raman, S. Designing a liveable compact city: physical forms of city and social life in urban neighbourhoods. Built Environ. 36, 63-80 (2010).

67. Golden, T. D., Veiga, J. F. \& Dino, R. N. The impact of professional isolation on teleworker job performance and turnover intentions: does time spent teleworking, interacting face-to-face, or having access to communication-enhancing technology matter? J. Appl. Psychol. 93, 1412-1421 (2008).

68. Doray, N. Cognitive Biases in Corporate Climate Action: How Industry Leaders are Mitigating Cognitive Bias in the Transition to a Low-Carbon Economy. PhD thesis, York Univ. (2019).

69. Mazur, C., Contestabile, M., Offer, G. J. \& Brandon, N. P. Assessing and comparing German and UK transition policies for electric mobility. Environ. Innov. Soc. Transit. 14, 84-100 (2015).

70. Wang, T. et al. Health co-benefits of achieving sustainable net-zero greenhouse gas emissions in California. Nat. Sustain. 3, 597-605 (2020).

71. Karlsson, M., Alfredsson, E. \& Westling, N. Climate policy co-benefits: a review. Clim. Policy 20, 292-316 (2020).

72. Klimaneutrales Deutschland 2045: Wie Deutschland seine Klimaziele schon vor 2050 erreichen kann (Prognos, Öko-Institut, Wuppertal-Institut, 2021) https://www.agora-energiewende.de/presse/neuigkeiten-archiv/ klimaneutralitaet-in-deutschland-bereits-2045-moeglich/ (2021).

73. Giallouros, G., Kouis, P., Papatheodorou, S. I., Woodcock, J. \& Tainio, M. The long-term impact of restricting cycling and walking during high air pollution days on all-cause mortality: health impact assessment study. Environ. Int. 140, 105679 (2020).

74. Ürge-Vorsatz, D., Herrero, S. T., Dubash, N. K. \& Lecocq, F. Measuring the co-benefits of climate change mitigation. Annu. Rev. Environ. Resour. 39, 549-582 (2014).

75. Dastrup, S. R., Zivin, J. G., Costa, D. L. \& Kahn, M. E. Understanding the solar home price premium: electricity generation and 'green' social status. Eur. Economic Rev. 56, 961-973 (2012).

76. Ramakrishnan, A. \& Creutzig, F. Status consciousness in energy consumption decisions: a systematic review. Environ. Res. Lett. 16, 053010 (2021).

77. Springmann, M. et al. Health-motivated taxes on red and processed meat: a modelling study on optimal tax levels and associated health impacts. PLoS ONE 13, e0204139 (2018).

78. Sulikova, S., van den Bijgaart, I., Klenert, D. \& Mattauch, L. Optimal Fuel Taxation with Suboptimal Health Choices Working Paper in Economics 794 (Univ. of Gothenburg, 2020); https://ideas.repec.org/p/hhs/gunwpe/0794. html

79. Kuhnhenn, K., Costa, L., Mahnke, E., Schneider, L. \& Lange, S. A Societal Transformation Scenario for Staying Below $1.5^{\circ} \mathrm{C}$ (Heinrich Böll Foundation and Konzeptwerk Neue Ökonomie, 2020); https://www.boell.de/en/2020/ 12/09/societal-transformation-scenario-staying-below-15degc

80. Niamir, L. et al. Assessing the macroeconomic impacts of individual behavioral changes on carbon emissions. Clim. Change 158, 141-160 (2020).

81. Ahl, A., Accawi, G., Hudey, B., Lapsa, M. \& Nichols, T. Occupant behavior for energy conservation in commercial buildings: lessons learned from competition at the Oak Ridge National Laboratory. Sustainability 11, 3297 (2019).

82. Institute for Global Environmental Strategies, Aalto University \& D-mat ltd 1.5-Degree Lifestyles: Targets and Options for Reducing Lifestyle Carbon Footprints (Institute for Global Environmental Strategies, 2019); https:// www.iges.or.jp/en/publication_documents/pub/technicalreport/en/6719/15_ Degree_Lifestyles_MainReport.pdf

83. Net Zero by 2050: From Whether to How (NECF, 2018); https:// europeanclimate.org/wp-content/uploads/2019/11/09-18-net-zero-by-2050from-whether-to-how.pdf

84. Mieux Vivre en Nord-Pas de Calais (Virage-énergie Nord-Pas de Calais, 2016); http://www.virage-energie.org/wp-content/uploads/2016/01/ Virage-\%C3\%A9nergie-NPdC_Rapport-complet-\%C3\%A9tude-mieux vivre_mars2016-1.pdf

85. Niamir, L., Ivanova, O. \& Filatova, T. Economy-wide impacts of behavioral climate change mitigation: linking agent-based and computable general equilibrium models. Environ. Model. Softw. 134, 104839 (2020).

86. Mastrucci, A. \& Rao, N. D. Bridging India's housing gap: lowering costs and $\mathrm{CO}_{2}$ emissions. Build. Res. Inf. 47, 8-23 (2019).

87. Mata, É., Kalagasidis, A. S. \& Johnsson, F. Contributions of building retrofitting in five member states to EU targets for energy savings. Renew. Sustain. Energy Rev. 93, 759-774 (2018).

88. Mata, É. et al. A map of roadmaps for zero and low energy and carbon buildings worldwide. Environ. Res. Lett. 15, 113003 (2020).
89. Ellsworth-Krebs, K., Reid, L. \& Hunter, C. J. Home comfort and 'peak household': implications for energy demand. Hous. Theory Soc. 38, 1-20 (2019).

90. Pomponi, F. et al. A novel method for estimating emissions reductions caused by the restriction of mobility: the case of the COVID-19 pandemic. Environ. Sci. Technol. Lett. 8, 46-52 (2021).

91. Brand, C., Dons, E. \& Anaya-Boig, E. The climate change mitigation effects of active travel. Preprint at Research Square https://doi.org/10.21203/ rs.3.rs-39219/v1 (2021).

92. Ivanova, D. et al. Quantifying the potential for climate change mitigation of consumption options. Environ. Res. Lett. 15, 093001 (2020).

93. Senbel, M., Giratalla, W., Zhang, K. \& Kissinger, M. Compact development without transit: life-cycle GHG emissions from four variations of residential density in Vancouver. Environ. Plan. A 46, 1226-1243 (2014).

94. Sheppard, C. J. R., Jenn, A. T., Greenblatt, J. B., Bauer, G. S. \& Gerke, B. F. Private versus shared, automated electric vehicles for U.S. personal mobility: energy use, greenhouse gas emissions, grid integration, and cost impacts. Environ. Sci. Technol. 55, 3229-3239 (2021).

95. Shared Mobility Simulations for Lyon (ITF, 2020); https://www.itf-oecd.org/ shared-mobility-simulations-lyon

96. Good to Go? Assessing the Environmental Performance of New Mobility (ITF, 2020); https://www.itf-oecd.org/good-go-assessing-environmentalperformance-new-mobility

97. Transition to Shared Mobility (ITF, 2017); https://www.itf-oecd.org/ transition-shared-mobility

98. Shared Mobility Simulations for Helsinki (ITF, 2017); https://www.itf-oecd $\mathrm{org} / \mathrm{sites} /$ default/files/docs/shared-mobility-simulations-helsinki.pdf

99. Shared Mobility: Innovation for Liveable Cities (ITF, 2016); https://www. itf-oecd.org/shared-mobility-innovation-liveable-cities

100. Ehrenberger, S. et al. Land transport development in three integrated scenarios for Germany - technology options, energy demand and emissions. Transp. Res. D Transp. Environ. 90, 102669 (2021).

101. Hou, F. et al. Comprehensive analysis method of determining global long-term GHG mitigation potential of passenger battery electric vehicles. J. Clean. Prod. 289, 125137 (2021).

102. Hampshire, K., German, R., Pridmore, A. \& Fons, J. Electric Vehicles from Life Cycle and Circular Economy Perspectives (electrive.com, 2018); https://www.electrive.com/study-guide/electric-vehicles-from-life-cycleand-circular-economy-perspectives/

103. Hill, G., Heidrich, O., Creutzig, F. \& Blythe, P. The role of electric vehicles in near-term mitigation pathways and achieving the UK's carbon budget. Appl. Energy 251, 113111 (2019).

104. Plötz, P., Funke, S. A., Jochem, P. \& Wietschel, M. $\mathrm{CO}_{2}$ mitigation potential of plug-in hybrid electric vehicles larger than expected. Sci. Rep. 7, 16493 (2017).

105. Clark, M. A. et al. Global food system emissions could preclude achieving the $1.5^{\circ}$ and $2^{\circ} \mathrm{C}$ climate change targets. Science 370, 705-708 (2020).

106. Makov, T., Shepon, A., Krones, J., Gupta, C. \& Chertow, M. Social and environmental analysis of food waste abatement via the peer-to-peer sharing economy. Nat. Commun. 11, 1156 (2020).

107. Poore, J. \& Nemecek, T. Reducing food's environmental impacts through producers and consumers. Science 360, 987-992 (2018).

108. Hiç, C., Pradhan, P., Rybski, D. \& Kropp, J. P. Food surplus and its climate burdens. Environ. Sci. Technol. 50, 4269-4277 (2016).

109. Semba, R. D. et al. Adoption of the 'planetary health diet' has different impacts on countries' greenhouse gas emissions. Nat. Food 1, 481-484 (2020).

110. Springmann, M. et al. Health and nutritional aspects of sustainable diet strategies and their association with environmental impacts: a global modelling analysis with country-level detail. Lancet Planet. Health $\mathbf{2}$, e451-e461 (2018)

111. Willett, W. et al. Food in the Anthropocene: the EAT-Lancet Commission on healthy diets from sustainable food systems. Lancet 393, 447-492 (2019).

112. Parodi, A. et al. The potential of future foods for sustainable and healthy diets. Nat. Sustain. 1, 782-789 (2018).

113. Hertwich, E., Lifset, R., Pauliuk, S. \& Heeren, N. Resource Efficiency and Climate Change: Material Efficiency Strategies for a Low-Carbon Future (IRP 2020); https://stg-wedocs.unep.org/bitstream/handle/20.500.11822/34351/ RECCR.pdf? sequence $=1$ \&is Allowed $=y$

114. Pauliuk, S. et al. Linking service provision to material cycles: a new framework for studying the resource efficiency-climate change (RECC) nexus. J. Ind. Ecol. 25, 260-273 (2021).

115. Tracking Industry 2020 (IEA, 2020); https://www.iea.org/reports/ tracking-industry-2020

116. Allwood, J. M. \& Cullen, J. M. Sustainable Materials: With Both Eyes Open (Cambridge Univ. Press, 2012).

117. Carruth, M. A., Allwood, J. M. \& Moynihan, M. C. The technical potential for reducing metal requirements through lightweight product design. Resour. Conserv. Recycl. 57, 48-60 (2011). 
118. Lausselet, C., Urrego, J. P. F., Resch, E. \& Brattebø, H. Temporal analysis of the material flows and embodied greenhouse gas emissions of a neighborhood building stock. J. Ind. Ecol. 25, 419-434 (2021).

119. Cooper, D. R., Skelton, A. C. H., Moynihan, M. C. \& Allwood, J. M. Component level strategies for exploiting the lifespan of steel in products. Resour. Conserv. Recycl. 84, 24-34 (2014).

120. Completing the Picture: How the Circular Economy Tackles Climate Change (Ellen MacArthur Foundation, 2019); https://www.

ellenmacarthurfoundation.org/assets/downloads/Completing_The Picture_How_The_Circular_Economy-_Tackles_Climate_Change_V3_26_ September.pdf

121. Material Efficiency in Clean Energy Transitions (IEA, 2019); https://www.iea. org/reports/material-efficiency-in-clean-energy-transitions

122. The Circular Economy - A Powerful Force for Climate Mitigation (Material Economics, 2018); https://materialeconomics.com/publications/thecircular-economy-a-powerful-force-for-climate-mitigation-1

123. Crijns-Graus, W., Yue, H., Zhang, S., Kermeli, K. \& Worrell, E. in Encyclopedia of Renewable and Sustainable Materials (eds Hashmi, S. \& Choudhury, I. A.) 377-388 (Elsevier, 2020).

124. Annual Review 2020 (IATA, 2020); https://www.iata.org/contentassets/ c81222d96c9a4e0bb4ff6ced0126f0bb/iata-annual-review-2020.pdf

125. Schäfer, A. W. et al. Technological, economic and environmental prospects of all-electric aircraft. Nat. Energy 4, 160-166 (2019).

126. Sharmina, M. et al. Decarbonising the critical sectors of aviation, shipping, road freight and industry to limit warming to $1.5-2{ }^{\circ} \mathrm{C}$. Clim. Policy 21, 455-474 (2021).

127. Bouman, E. A., Lindstad, E., Rialland, A. I. \& Strømman, A. H. State-of-the-art technologies, measures, and potential for reducing GHG emissions from shipping-a review. Transp. Res. D Transp. Environ. 52, 408-421 (2017).

128. McKinnon, A. Decarbonizing Logistics: Distributing Goods in a Low Carbon World (Kogan Page Publishers, 2018).

129. Decarbonising Maritime Transport (ITF, 2018); https://www.itf-oecd.org/ decarbonising-maritime-transport

130. Roy, J. et al. in IPCC Special Report on Global Warming of $1.5^{\circ} \mathrm{C}$ (eds Masson-Delmotte, V. et al.) Ch. 5 (WMO, 2018); https://www.ipcc.ch/site/ assets/uploads/sites/2/2019/05/SR15_Chapter5_Low_Res.pdf

131. O’Reilly, J., Isenhour, C., McElwee, P. \& Orlove, B. Climate change: expanding anthropological possibilities. Annu. Rev. Anthropol. 49, 13-29 (2020).
132. Creutzig, F. Limits to liberalism: considerations for the Anthropocene. Ecol. Econ. 177, 106763 (2020).

133. Mattauch, L., Hepburn, C. \& Stern, N. Pigou Pushes Preferences: Decarbonisation and Endogenous Values Climate Change Economics and Policy Working Paper 346/Grantham Research Institute on Climate Change and the Environment Working Paper 314 (London School of Economics and Political Science, 2018); https://www.lse.ac.uk/granthaminstitute/wpcontent/uploads/2018/12/working-paper-314-Mattauch-et-al.pdf

134. Hawkes, C. et al. Smart food policies for obesity prevention. Lancet 385, 2410-2421 (2015).

135. Larcom, S., Rauch, F. \& Willems, T. The benefits of forced experimentation: striking evidence from the London underground network. Q. J. Econ. 132, 2019-2055 (2017).

136. Bamberg, S., Rölle, D. \& Weber, C. Does habitual car use not lead to more resistance to change of travel mode? Transportation 30, 97-108 (2003).

137. Weinberger, R. \& Goetzke, F. Unpacking preference: how previous experience affects auto ownership in the United States. Urban Stud. 47, 2111-2128 (2010).

138. Grinblatt, M., Keloharju, M. \& Ikäheimo, S. Social influence and consumption: evidence from the automobile purchases of neighbors. Rev. Econ. Stat. 90, 735-753 (2008).

139. Baranzini, A., Carattini, S. \& Péclat, M. What Drives Social Contagion in the Adoption of Solar Photovoltaic Technology GRI Working Paper 270 (Grantham Research Institute on Climate Change and the Environment, 2017); https://ideas.repec.org/p/lsg/lsgwps/wp270.html

140. Lanz, B., Wurlod, J.-D., Panzone, L. \& Swanson, T. The behavioral effect of pigovian regulation: evidence from a field experiment. J. Environ. Econ. Manage. 87, 190-205 (2018).

141. Rivers, N. \& Schaufele, B. Salience of carbon taxes in the gasoline market. J. Environ. Econ. Manage. 74, 23-36 (2015)

142. Andersson, J. J. Carbon taxes and $\mathrm{CO}_{2}$ emissions: Sweden as a case study. Am. Econ. J. Econ. Policy 11, 1-30 (2019).

143. Stern, N. Why Are We Waiting? The Logic, Urgency, and Promise of Tackling Climate Change (MIT Press, 2015).

144. Brulle, R. J. \& Aronczyk, M. in Routledge Handbook of Global Sustainability Governance (eds Kalfagianni, A. et al.) Ch. 17 (Routledge, 2019).

Publisher's note Springer Nature remains neutral with regard to jurisdictional claims in published maps and institutional affiliations.

(c) The Author(s), under exclusive licence to Springer Nature Limited 2021 


\section{Methods}

Overview. A mixed-and multi-methodology framework has been designed and used to identify and assess demand-side mitigation strategies and their impacts on GHG emissions and human well-being (Extended Data Fig. 3). In the following we explain the steps, methods and validity and reliability in detail.

Identifying and assessing demand-side mitigation options. Two workshops were designed (April and October 2019) with the objective of defining, structuring and evaluating demand-side mitigation options (for the third workshop see Assessing effects on well-being). The first workshop was held in person in April 2019 with the participation of 36 experts, including sectoral (building, food, transport, urban and industry) experts and energy demand-side, technology, finance, well-being and social scientists. Experts were academics with expertise in climate change mitigation and well-being evaluation (for example, via the cobenefit approach) in at least one sector, as demonstrated by publication track records. The first workshop aimed to brainstorm and identify demand-side mitigation options using the categorization of the ASI concept. All discussions (two broad rounds and 11 subgroup discussions) were documented and shared with participants. As a next step, experts in the form of five sectoral teams further identified and structured demand-side options within the ASI framework (Extended Data Fig. 3). Sectoral teams searched, screened and coded the relevant literature based on their expert knowledge of this (for additional systematic confirmation and supplementation, see below) from the sector-specific scenario and option literature, reduction potential estimates and ranges (Literature databases). Sectoral teams reported back their findings before the second workshop for an internal review process (Extended Data Fig. 3)

The second workshop was also held in person, in October 2019 with 30 experts, including sectoral team experts and reviewers (Extended Data Fig. 3). The demand-side mitigation options were further discussed in this workshop, following feedback from the internal review team and workshop co-organizers. As a result, with the help of these two in-person workshops (April and October 2019), the sectoral team's extensive studies and an internal review, three or four comprehensive demand-side strategies were selected for each sector. In this process, several factors were considered: (1) being comprehensive (as an umbrella of several options, which might differ over sectors); (2) categorization within the ASI framework; and (3) relevant potential in mitigating climate changes. For example, we identified several demand-side mitigation options in the food sector, including changes in diet, shift to regional, seasonal and organic consumption, reduction in food consumption and improvement in packing technology. We selected 'animal-free proteins' and 'food waste' as the top two, simply due to their clear links to mitigation and high impacts. We decided on the third option as 'overconsumption' because of its very strong impact on well-being through its health effects.

We organized demand-side mitigation strategies according to both sector (building, transport, food, urban, industry) and mitigation strategy (ASI) (summary in Table 1, full details in Supplementary Table 1). Demand-side mitigation potentials were assessed through a comprehensive literature review and in several stages. The lower and upper boundaries and centre were identified and reportedin rounded numbers-based on both expert judgement and what was presented in the literature. Supplementary Table 1 shows the internal review, together with well-being tables, conducted by the team of experts of whom nine members had relevant sectoral and/or social science expertise (Extended Data Fig. 3).

Assessment of synergies and trade-off between SDGs and energy demand/ supply solutions. In the SR1.5 IPCC Report, the assessment presents positive and negative links of individual mitigation options with each of the SDGs according to their relative strength and level of confidence. Strengths are scored between 0 and 3 , and confidence levels are represented by one to four asterisks. Using this detailed information, we derive here the ratio of positive to negative links in more aggregated form, as detailed in the following steps. First, we counted confidence-level-wise positive and negative links for sectors. In the second step we summed these using the numerical values of scores of strengths for each of the links. Then, in the third step, aggregate values for demand and supply categories were calculated using corresponding confidence levels as weights. For example, for the building sector, the total count of positive links/synergies for very low confidence $\left(^{*}\right.$ ) was 13 , for low confidence $\left.{ }^{* *}\right) 15$, for medium confidence $\left.{ }^{* * *}\right) 24$, for high confidence ${ }^{(* * *)} 15$ and total trade-offs were $0,2,4$ and 0 , respectively for the different levels of confidence. Each of these values was weighted according to confidence levels as applicable $(1,2,3,4)$, which yields 175 for a weighted positive sum and 16 for a weighted negative sum; finally the ratio between these two weighted sums was calculated as $\left(\frac{175}{16}=10.9\right)$. This procedure was followed for each of the sectors.

Measurement of well-being. The literature on human well-being is complicated by varying definitions and overlapping terminology. Terms such as 'human needs', 'well-being,' 'subjective well-being,' 'happiness', 'welfare' and 'quality of life' are often used interchangeably and imprecisely. A widely perceived divide separates well-being concepts into three broad camps: preference satisfaction, hedonic and eudaimonic positions ${ }^{145,146}$, with diverging implications for climate change mitigation ${ }^{12,13}$. The preference satisfaction position, as introduced above, takes citizens' preferences satisfaction as constituting well-being and is therefore in some form committed to the view that whatever people choose makes them better off. It is hence closely related to the association of higher income with higher well-being, and typically measures the degree to which preferences are satisfied in market transactions and beyond markets as income. Second, in the hedonic view, well-being is a matter of maximizing individuals' happiness, or health. It can be measured, for example, via 'life satisfaction' and 'happiness' surveys and is often interpreted as the subjective perception of well-being conditions in society. A great deal of research examines the individual and social determinants of variation in happiness, health and life satisfaction. This approach builds on utilitarian philosophy.

A third category of eudaimonic concepts focuses on objective conditions and actions that underpin well-being. This constitutes a large family of theories, most notably on capabilities ${ }^{14,147}$, human needs $s^{15,148-150}$, multi-dimensional poverty ${ }^{151}$ and so forth. The core claim is to identify and separate a universal set of basic conditions that are required by all humans for a good life, from their satisfiers, which can be culturally and individually diverse. We adopt the eudaimonic position on well-being by the analysis that follows, for two reasons. First, a eudaimonic approach is consistent with changing preferences, as the focus is on substantive conditions of a good life that are independent of changing preferences (nonetheless, even if preferences are changing, demand-side solutions could also be evaluated by approaches that account for fundamental preferences ${ }^{151-153}$ ). Second, a eudaimonic approach is largely under-represented in the context of climate change mitigation, as the current literature evaluating climate policies and measures nearly exclusively takes an implicit or explicit given preference approach, often shortcut with economic growth metrics.

Despite the very diverse nature of the literature on eudaimonic well-being, broad surveys have centred on a number of core conditions that achieve consensus across epistemic divides ${ }^{12,154}$. The constituents of eudaimonic well-being include essential material conditions of a good life, such as food and energy, but also clean water, sanitation, air quality and social dimensions, such as social cohesion and political stability (Extended Data Fig. 1). Importantly, these constituents are nearly all reflected in the SDGs (Extended Data Fig. 1) and thus have political legitimacy among nations worldwide.

During the second workshop (October 2019) the well-being concept was presented by workshop co-organizers, and the potential metrics, links to SDGs and demand-side mitigation strategies were discussed. Workshop co-organizers proposed a matrix of sectoral demand-side mitigation options and well-being dimensions (used to organize Supplementary Tables 3-7).

Assessment of the effects on well-being. As a result of the second workshop, workshop co-organizers designed and developed an online platform for sectoral teams, providing a space for each to code the effects of demand-side options on well-being in 19 different categories, presented in Extended Data Fig. 1. In addition, it allowed them to see the coding and progress in other sectors. We used the sectoral teams' judgement and evaluation (Extended Data Fig. 3), and a concurrent literature search on 306 combinations of well-being and demand-side measures used, to create Supplementary Tables 3-7 and Fig. 3 (Literature databases).

While not all combinations were judged relevant, we supported judgements for existing relationships between demand-side options and well-being with 604 references. Experts identified potentially relevant publications through a mixture of their in-depth knowledge of the field and targeted keyword-based queries in relevant bibliographic databases (Literature databases). In addition, to develop our key findings, the teams of experts evaluated the associated evidence, agreement and confidence levels of each entry. Confidence in the validity of a finding was based on the type, amount, quality and consistency of evidence (for example, mechanistic understanding, theory, data, models, expert judgement), and the degree of agreement (Supplementary Tables 3-7). Furthermore, all steps were subjected to three rounds of internal review including by social scientists and well-being-, sector- and domain-specific experts (Supplementary Tables 3-7). To also mirror the state of the literature, reflecting highly different literature bases on the combination of well-being dimensions and demand-side measures, and to represent uncertainty in interpretation of the literature, we also coded for the confidence of well-being impacts in all 306 combinations (Extended Data Fig. 2).

Within our online platform, five comprehensive sectoral tables were designed: building, food, transport, urban and industry (Supplementary Tables 3-7). The potential of each demand-side mitigation strategy on well-being dimensions was evaluated by the teams of experts based on the existing literature and the experts scientific judgement. The impact was coded as $-3,-2,-1,0,+1,+2$ and +3 , denoting highly negative to highly positive impact. In addition, to develop our key findings the teams of experts evaluated the associated evidence, agreement and confidence levels of each entry. Confidence in the validity of a finding was based on the type, amount, quality and consistency of evidence (for example, mechanistic understanding, theory, data, models, expert judgement) and the degree of agreement.

The level of evidence (limited, medium or robust) and degree of agreement (low, medium or high) represented by 国 and (-), respectively, in Supplementary Tables 3-7, were evaluated by sectoral expert teams based on the amount, quality and consistency of evidence. The level of confidence was expressed using five qualifiers: very low, low, medium, high and very high, represented by asterisks in 
Supplementary Tables 3-7. It synthesizes the expert teams' judgement about the validity of findings as determined through evaluation of evidence and agreement.

Five sectoral teams reported back their assessments before the third workshop for an internal review process (Extended Data Fig. 3). In the second round of internal review, at least two reviewers (members of the author team), based on their expertise, were assigned to look at three or four well-being dimensions over five sectors, and to review and evaluate the sectoral teams' assessments. Internal reviewers reported back their comprehensive evaluation with detailed notes and suggestions to the sectoral teams (Extended Data Fig. 3).

In April 2020 the third workshop was held virtually (via Zoom), where we hosted 45 participants (Extended Data Fig. 3). By presenting a preliminary version of Fig. 1, co-organizers discussed demand-side mitigation options and potentials in the context of ASI over five sectors. Links to human well-being and SDGs were explained by presentation of a preliminary version of Fig. 3 (summary and simplified version of Supplementary Tables 3-7). In addition, a major discussion was created on how to deal with and measure cross-sectors and cross-cutting issues. The third workshop guided us to further assess, re-evaluate and recode mitigation potentials and well-being measures, and thereby further develop Table 1 and Supplementary Tables 3-7 (Extended Data Fig. 3). Experts used our comprehensive literature (about 54,000 documents) database to assess the relevant literature, responded to internal review comments and discussed crucial points with internal reviewers bilaterally. The revised versions of Table 1 and Supplementary Tables 3-7 were again reviewed by five experts (Extended Data Fig. 3). The last few comments and suggestions were implemented in Table 1 and Supplementary Tables 3-7 in cooperation with the sectoral teams.

Literature databases. In this study, three techniques/types of literature review were used over various stages. First, sectoral teams used the narrative and argumentative literature review techniques to review the relevant literature on demand-side mitigation options and potentials. By narrowing down search queries, each sectoral team scanned $>500$ relevant publications and assessed and coded sectoral mitigation options and their impact on GHG emissions (Supplementary Table 1), as well as coding well-being impacts (Supplementary Tables 3-7). Our internal reviewers used an argumentative review technique to evaluate expert judgements and examine confidence levels (Supplementary Tables 3-7). Only through these techniques were $>1,000$ papers screened.

To improve the validity and reliability of this study and avoid bias in the experts' opinions, with the help of a systematic literature survey and machine learning techniques a comprehensive literature database on demand-side mitigation strategies and well-being was designed. First, queries were designed separately for each of the 17 demand-side mitigation strategies and 19 dimensions of well-being (Supplementary Table 2a). We extracted the title, author, year and abstract of 54,000 documents from Web of Science, and automatically compiled lists of studies matching the 360 combinations of mitigation strategies and dimensions of well-being. This database was used by the experts-the sectoral teams and internal reviewers - to assess the potential of demand-side mitigation strategies and impacts on well-being. We set up the interactive database in which, by clicking on individual cells, experts were guided to the associated dataset (Supplementary Table 2b).

\section{Data availability}

All data used for Figs. 1 and 3 are fully presented in the Extended data. The literature database $\mathrm{e}^{155}$ is openly available: https://doi.org/10.5281/zenodo.5163965

\section{References}

145. Fleurbaey, M. \& Blanchet, D. Beyond GDP: Measuring Welfare and Assessing Sustainability (Oxford Univ. Press, 2013).
146. Roger, C. Well-being in The Stanford Encyclopedia of Philosophy (ed. Zalta, E. N.) (The Metaphysics Research Lab, 2008); http://plato.stanford.edu/ archives/win2008/entries/well-being

147. Nussbaum, M. Creating Capabilities (Harvard Univ. Press, 2011).

148. Doyal, L. \& Gough, I. in Mixed Economies in Europe (eds Blaas, W. \& Foster, J.) 178-199 (Edward Elgar Publishing, 1993).

149. Gough, I. Heat, Greed and Human Need: Climate Change, Capitalism and Sustainable Wellbeing (Edward Elgar Publishing, 2017)

150. Alkire, S. in Wellbeing in Developing Countries (eds Gough, I. \& Allister McGregor, J.) 93-108 (Cambridge Univ. Press, 2007).

151. Von Weizsäcker, C. C. Notes on endogenous change of tastes. J. Econ. Theory 3, 345-372 (1971).

152. Fleurbaey, M. \& Tadenuma, K. Universal social orderings: an integrated theory of policy evaluation, inter-society comparisons, and interpersonal comparisons. Rev. Econ. Stud. 81, 1071-1101 (2014).

153. Mattauch, L. \& Hepburn, C. Climate policy when preferences are endogenous-and sometimes they are. Midwest Stud. Philos. 40, 76-95 (2016).

154. Lissner, T. K., Reusser, D. E., Lakes, T. \& Kropp, J. P. A systematic approach to assess human wellbeing demonstrated for impacts of climate change. Change Adapt. Socioecol. Syst. 1, 98-110 (2014).

155. Creutzig, F. \& Niamir, L. Demand-side solutions to climate change mitigation consistent with high levels of wellbeing. Preprint at Zenodo https://doi.org/10.5281/zenodo.5163965 (2020).

\section{Acknowledgements}

We thank our workshop participants for their contribution and feedback. L.N. and F.C., J.C.M. and M.C., and W.F.L. acknowledge funding from Bundesministerium für Bildung und Forschung (Federal Ministry of Education and Research) project number 01LG1806A, 03SFK5J0 and 01LG1910A respectively. L.M. acknowledges funding from the Robert Bosch Foundation.

\section{Author contributions}

F.C. and L.N. designed the framework of this study and led the process and writing. The sectoral teams: Y.S., E.M.M. and S.M. (building); A.L., J.D. and L.N. (food); M.F., S.B.N. and F.C. (transport); M.P., X.B. and D.U.V. (urban); and J.C., S.d.l.R.d.C. and E.M. (industry). L.M., W.F.L., A.G., E.M., J.C.M., Y.M., P.P., J.R., L.S., J.S., F.C. and L.N were the internal review team. M.C. and L.N. designed and supported the systematic literature survey. S.S. and J.R. provided trade-offs between SDGs and demand/supply solutions (Fig. 2). L.N. and F.C. designed Figs. 1 and 3. All authors contributed to data collection, analysis and writing.

\section{Competing interests}

The authors declare no competing interests.

\section{Additional information}

Extended data is available for this paper at https://doi.org/10.1038/s41558-021-01219-y.

Supplementary information The online version contains supplementary material available at https://doi.org/10.1038/s41558-021-01219-y.

Correspondence and requests for materials should be addressed to Felix Creutzig.

Peer review information Nature Climate Change thanks Claire Hoolohan, Mari Martiskainen and the other, anonymous, reviewer(s) for their contribution to the peer review of this work.

Reprints and permissions information is available at www.nature.com/reprints. 


\begin{tabular}{|c|c|c|}
\hline Well-being dimension & SDGs & Potential metric and definition \\
\hline Food & 2 & access to sufficient and safe nutrition \\
\hline Water & 6 & access to adequate and clean drinking water \\
\hline Air & 7,11 & $\begin{array}{l}\text { exposure to dangerous concentrations; pollutants both indoor and } \\
\text { outdoor }\end{array}$ \\
\hline Health & 3 & access to health services; physical and mental health ; obesity \\
\hline Sanitation & 6 & access to adequate sanitation; waste and sewage management \\
\hline Energy & 7 & $\begin{array}{l}\text { ability to attain a socially and materially necessitated level of energy } \\
\text { services (often related to access to electricity); access to affordable, } \\
\text { reliable and sustainable fuels (electricity); renewable and clean } \\
\text { electricity }\end{array}$ \\
\hline Shelter & 11 & access to accommodation; affordable housing market \\
\hline Mobility & 11 & $\begin{array}{l}\text { ability to access key other services physically in a safe and affordable } \\
\text { manner; access to safe walking and cycling infrastructures, and to } \\
\text { public transport }\end{array}$ \\
\hline Education & 4 & $\begin{array}{l}\text { education for all ; access to education and material ; knowledge and } \\
\text { information }\end{array}$ \\
\hline Communication & & $\begin{array}{l}\text { ability to make human connections with and without personal } \\
\text { meetings; access to information and entertainment }\end{array}$ \\
\hline Social protection & $1,2,8,10$ & $\begin{array}{l}\text { community, social insurance, social assistance, and labor markets that } \\
\text { enhance people's capacity to manage economic and social risks, e.g. } \\
\text { unemployment, exclusion, sickness, disability and old age. }\end{array}$ \\
\hline Participation & $5,10,16$ & democratic rights (voting, association, etc.) \\
\hline Personal Security & 5,16 & exposed to homicide, crime, war/state violence \\
\hline Social cohesion & 10,16 & $\begin{array}{l}\text { social trust; bottom-up initiatives; reduce inequality; sense of } \\
\text { usefulness }\end{array}$ \\
\hline Political stability & 11,16 & trust in politicians; good governance; quality of governance \\
\hline Economic stability & 8 & not having to fear unexpected expenses; access to jobs \\
\hline Material provision & 9,12 & $\begin{array}{l}\text { ability to access wellbeing services which are derived from materials; } \\
\text { provision of adequate industrial capacity; access to infrastructure }\end{array}$ \\
\hline Supply side / incumbents & 8,12 & $\begin{array}{l}\text { upstream effects of demand-side measures on upstream production } \\
\text { systems (e.g.: compact cities make reduce demand for cars, increase } \\
\text { demand for shared mobility) }\end{array}$ \\
\hline
\end{tabular}

Extended Data Fig. 1 | Constituents of wellbeing and their relationship to SDGs. 


\begin{tabular}{|c|c|c|c|c|c|c|c|c|c|c|c|c|c|c|c|c|c|c|}
\hline & SDGs & 2 & 6 & 7,11 & 3 & 6 & 7 & 11 & 11 & 4 & & $1,2,8,10$ & $5,10,16$ & 5,16 & 10.16 & 11,16 & 8 & 9,12 \\
\hline Sectors & $\begin{array}{l}\text { Mitigation strategies / Wellbeing dimensions } \\
\begin{array}{|l|l|} & \text { Very high confidence level } \\
\text { High con fid ence level } \\
\text { Medium confidence level } \\
\text { Low confidence level } \\
\\
\text { Very low confidence level }\end{array} \\
\end{array}$ & "ఃّ & 离 & 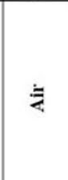 & 竎 & 竞 & $\stackrel{\vec{b}}{\stackrel{b}{u}}$ & 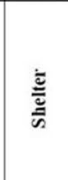 & $\begin{array}{l}\text { 訔 } \\
\text { 흘 }\end{array}$ & 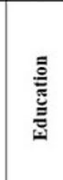 & 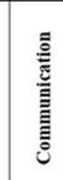 & 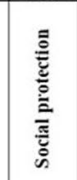 & 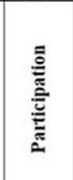 & 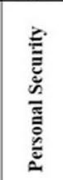 & 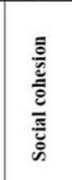 & 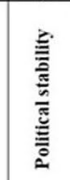 & 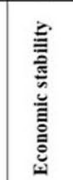 & 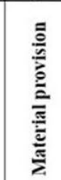 \\
\hline \multirow{3}{*}{ 总 } & Sufficiency & $\begin{array}{l}{[\cdot 1]} \\
\star \star \star *\end{array}$ & $\ldots \ldots$ & 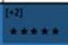 & ....... & [-1] & 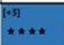 & {$\left[\begin{array}{lll}{[+1]} \\
*\end{array}\right.$} & [1+1] & t+1] & {$\left[\begin{array}{l}{[* 2]} \\
\star \star \star \star\end{array}\right.$} & {$\left[\begin{array}{l}{[+1]} \\
\star \star\end{array}\right.$} & $\begin{array}{l}{[\cdot 01]} \\
\star \star \star\end{array}$ & & *N. & & {$[-21$} & 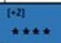 \\
\hline & Efficiency & $\stackrel{[-2]}{*}$ & {$[-2]$} & $\begin{array}{l}{[0,-\pi]} \\
\cdots * * *\end{array}$ & 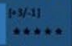 & {$[\cdot 1]$} & {$\left[\begin{array}{ll}* 1 \\
* \ldots * *\end{array}\right.$} & 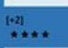 & & {$[* 1]$} & {$[\because 1]$} & & {$\left[\begin{array}{ll}{[-1]} \\
\cdots * *\end{array}\right.$} & 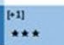 & 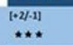 & & & {$[-1 ; z / 1]$} \\
\hline & Lower carbon and renewable energy & 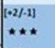 & $\mid[-2 / \lambda \mid$ & $\stackrel{1+x)}{\ldots \ldots \ldots *}$ & (1.3) & & 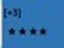 & {$\left[\begin{array}{lll}{[0]} \\
* \star *\end{array}\right.$} & {$[0.1]$} & {$[* 1]$} & $\stackrel{[\bullet 2]}{\star \star \star \star}$ & & 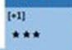 & 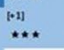 & $[-2 / 2]]$ & & & {$[102]$} \\
\hline \multirow{3}{*}{ Бั } & Food waste & {$[+1]$} & 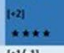 & 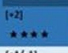 & {$[+2]$} & $\stackrel{[10]}{* *}$ & & & & & {$[\stackrel{[+1]}{\cdots}$} & $[1]+1]$ & $\left.{ }^{[+1]}\right]$ & & & {$[?[1]$} & $\stackrel{[\cdot 0]}{*}$ & \\
\hline & Over-consumption & {$[\cdot 1]$} & $\stackrel{[\cdot 1 / 1]}{\star}$ & {$[\cdot[1 / \cdot 1]$} & $\ldots$ & & {$[0.1 /-1]$} & & & & & & {$[\cdot 2]$} & & & {$[?[1]$} & & \\
\hline & Animal free protein & {$[-1]$} & 1021 & $[0]\}$ & {$[\because]$} & & & & & & {$\left[\begin{array}{ll}{[-1]} \\
\star \star \star \star\end{array}\right.$} & & {$[-\infty \mid]$} & & {$[-1]$} & {$[\cdot 1]$} & & \\
\hline \multirow{4}{*}{ 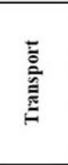 } & Teleworking and online education system & $\mid \cdot 2]$ & & 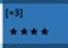 & [1] $\ldots$ & & {$[2]$} & 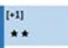 & {$[+3 \mid$} & {$[1]$} & {$[-2] \mid \cdots *$} & $\ldots$ & 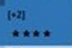 & $\frac{|-2|}{1-\ldots \ldots *}$ & 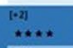 & $\ldots$ & $\stackrel{[\cdot 2]}{* * *}$ & \\
\hline & Non-motorized transport & $\mid+1]$ & $\stackrel{[\cdot 1]}{*}$ & & $[-\ldots]\}$ & & $\ldots$ & & & {$[-1]$} & {$[\cdot \ldots]$} & {$[\cdot[]$} & 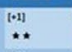 & $|x| \cdots$ & 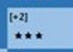 & $\left.{ }^{[2]}\right]$ & $\stackrel{[-1]}{\cdots *}$ & \\
\hline & Shared mobility & {$\left[\begin{array}{l}* 11 \\
\cdots *\end{array}\right.$} & & $\stackrel{[+3]}{* * *}$ & & & {$[0.1]$} & & & & 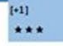 & $\begin{array}{l}{[02]} \\
\ldots * *\end{array}$ & $\stackrel{[\cdot 1]}{* * *}$ & 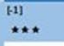 & ...... & 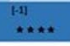 & $\begin{array}{l}{[.2]} \\
\cdots \cdots+.\end{array}$ & [.2] \\
\hline & Evs & $\stackrel{[-2]}{\cdots}$ & & {$[-2]$} & 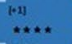 & [1+1] & {$[+2]$} & & 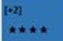 & & & & 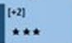 & & & & & {$[-1]$} \\
\hline \multirow{4}{*}{ 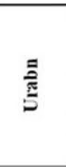 } & Compact city & $\mid \begin{array}{l}\mid+2 /-1] \\
\cdots \cdots\end{array}$ & $\stackrel{[+2]}{* *}$ & 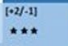 & {$\left[\begin{array}{l}[-3 / 1] 1] \\
* * \cdots\end{array}\right.$} & {$\left[\begin{array}{lll}{[1]} \\
* *\end{array}\right.$} & 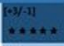 & \#.+* & {$[\cdot 3]$} & & {$\left[\begin{array}{l}{[-1 / 1 / 3]} \\
* * *\end{array}\right.$} & $\stackrel{\mid-2]}{* *}$ & $\stackrel{[-1]}{* *}$ & 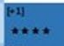 & $\ldots+\infty$ & & 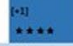 & $\stackrel{[\cdot]]}{* *}$ \\
\hline & Circular and shared economy & 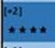 & $\stackrel{1+2]}{* * *}$ & $\stackrel{1 \cdot 2]}{* \ldots *}$ & $\stackrel{1+2]}{\cdots *}$ & & 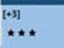 & 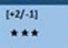 & H..... & {$[+1]$} & {$[\cdot \ldots$} & $\stackrel{[+2]}{\cdots *}$ & $\stackrel{[\cdot]]}{* \cdots *}$ & $|+2|$ & {$[+2]$} & $\stackrel{[+1]}{\cdots}$ & $\stackrel{[-2]}{\star *}$ & 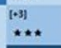 \\
\hline & Systems approach in urban policy and practice & {$\left[\begin{array}{ll}{[-1]} \\
\cdots\end{array}\right.$} & $\stackrel{[+2]}{\cdots}$ & $\stackrel{[\cdot 2]}{\ldots}$ & $\stackrel{[\cdot 3]}{\ldots . .}$ & $\stackrel{[+1]}{* * *}$ & {$[\cdot 3]$} & $\stackrel{\mid \cdot z]}{\cdots}$ & {$[+* 3$} & & $\stackrel{[+1]}{*}$ & $\stackrel{[1]}{* *}$ & 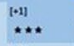 & $\stackrel{[+2]}{*}$ & $\stackrel{[+1]}{* *}$ & & $\stackrel{[+]]}{* *}$ & $\cdots$ \\
\hline & Nature based solutions & 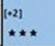 & {$\left[\begin{array}{ll}10 \pi \\
\ldots \ldots \ldots\end{array}\right.$} & 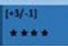 & pos & {$[1: \cdots]$} & {$[\cdot 2]$} & 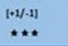 & $\stackrel{[+2]}{* * *}$ & {$[-2]$} & & $\stackrel{[\bullet 2]}{* *}$ & {$[* 3]$} & {$[* 1]$} & 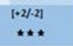 & & {$\left[\begin{array}{ll}{[0]} \\
* * *\end{array}\right.$} & 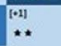 \\
\hline \multirow{5}{*}{ 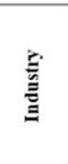 } & Using less material by design & 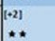 & {$[\cdot 2]$} & {$[\cdot 3]$} & {$[\cdot 2]$} & 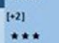 & F & 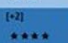 & {$[\cdot 2]$} & 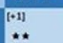 & $\stackrel{[+2]}{* \star \star}$ & $\stackrel{[+1]}{\star \star}$ & $\stackrel{[-1]}{* \star *}$ & {$[+2]$} & {$[+1]$} & {$[1+1]$} & {$[\cdot 2]$} & {$[-3]$} \\
\hline & Product life extension & {$\left[\begin{array}{c}1-2] \\
\ldots *\end{array}\right.$} & {$[1 \cdot 2]$} & {$[+3]$} & {$[\cdot 2]$} & {$[-2]$} & 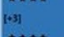 & {$[+2]$} & {$[\cdot 2]$} & {$[+1]$} & {$[\cdot 2]$} & {$[+1]$} & (1) & {$[1 ;]$} & {$[\cdot 1]$} & {$[+1]$} & {$[\cdot 2]$} & {$[13]$} \\
\hline & Eneroy Efficiency & {$[-2]$} & $\begin{array}{lll}* * 2 * \\
1 \cdot 2]\end{array}$ & $\cdots+\cdots$ & $\ddot{* * 1]}$ & $\stackrel{* * *}{[+2]}$ & & $\ldots * \ldots *$ & $\ldots * * *$ & $\underset{i+2]}{* *}$ & $\underset{[+2]}{* * *}$ & $\stackrel{* *}{[-2]}$ & $\mid * * * *$ & $* *$ & ** & $\stackrel{* *}{[+1]}$ & $\stackrel{\ldots *}{[-2]}$ & $\ddot{* *}$ \\
\hline & Energy Efliciency & $* *$ & $\star \cdots *$ & $\cdots$ & ** & $\ddot{* * *}$ & $\cdots \cdots$ & $\stackrel{* \ldots *}{[+2]}$ & $* * * *$ & $* *$ & $* * \star$ & $\ldots *$ & $\ldots * *$ & $* *$ & & $* *$ & $* * *$ & $* *$ \\
\hline & Circular economy & $\ldots \ldots$ & $\star * *$ & $\ldots * *$ & {$[* 1]$} & $\star \star \star \star *$ & $\ldots \ldots$ & $\ldots \ldots * *$ & (*... & $* *$ & $\stackrel{[+2]}{\ldots * *}$ & {$[\stackrel{[+2]}{* *}$} & {$[+2]$} & ** & $\stackrel{[+1]}{* \star}$ & & {$[* 2]$} & {$\left[\begin{array}{lll}{[\cdot 3]} \\
* \star\end{array}\right.$} \\
\hline
\end{tabular}

Extended Data Fig. 2 | Confidence of assessment in demand-side options/wellbeing rating, based on the state of the literature. Detailed data underpinning the assessment is reported in Supplementary Tables 3-7. 


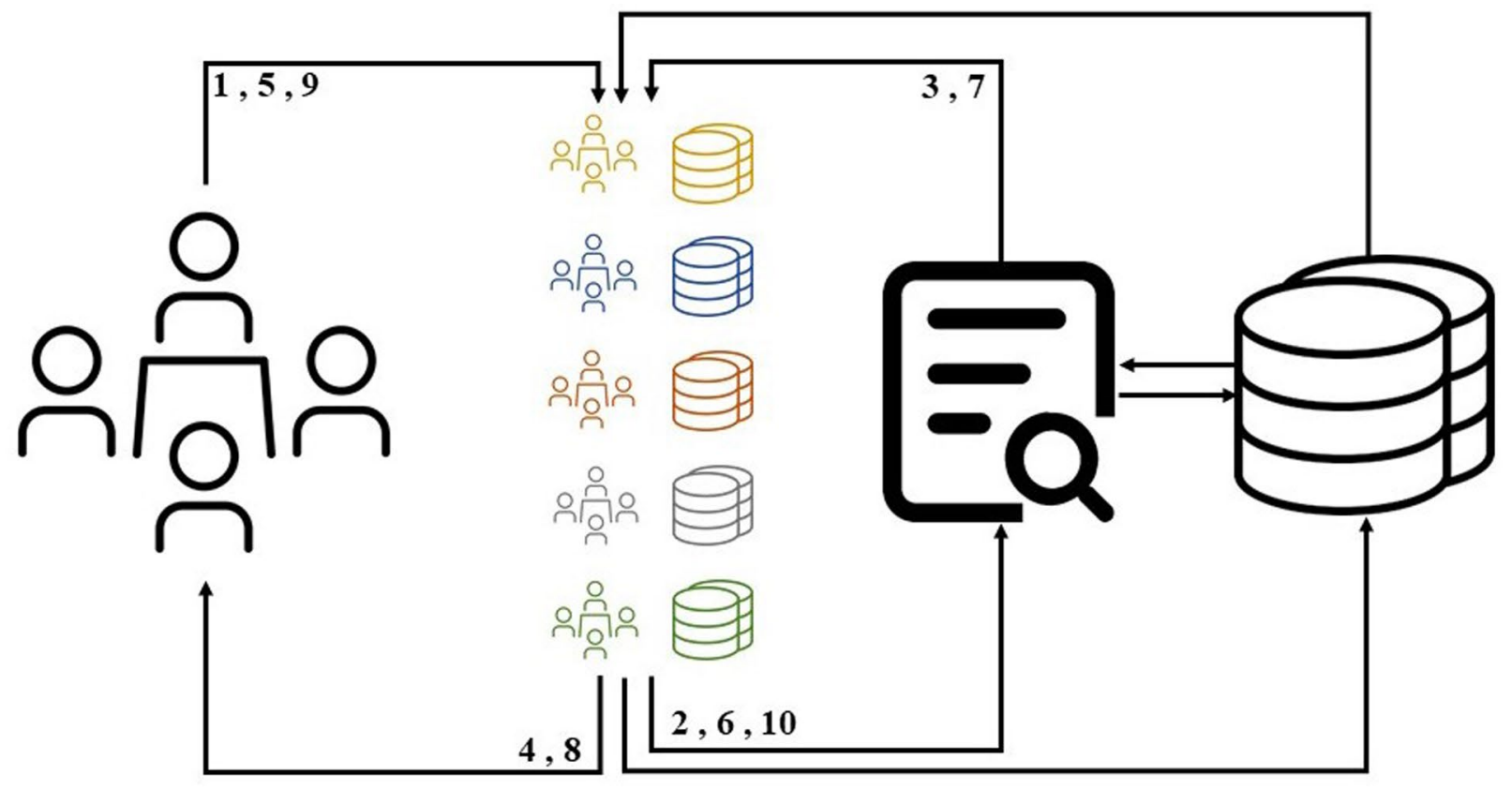

Three workshops $(1,5,9)$ participants: $30-45$ (including sectoral experts, social scientists, wellbeing experts)

\section{Sectoral teams} Building (3 experts); Food (3 experts); Transport (3 experts); Urban (3 experts); Industry (3 experts)
Three internal reviews $(2,6,10)$ reviewer team (including sectoral experts, social scientists, wellbeing experts) R\#1 (3 experts) R\#2 (9 experts) R\#3 (4 experts)
Literature Databases argumentative literature review narrative literature review systematic literature review

Extended Data Fig. 3 | Demand-side mitigation options and wellbeing potentials mixed- and multi-methods framework. workshop/meeting icon source: by Maxim Kulikov (CC BY 3.0) via Wikimedia Commons. Available from: https://commons.wikimedia.org/wiki/File:Noun_Project_Business_Meeting_ icon_1150615.svg. 